\title{
Neuronal and Psychophysical Sensitivity to Motion Signals in Extrastriate Area MST of the Macaque Monkey
}

\author{
Simona Celebrinia+ and William T. Newsome \\ Department of Neurobiology, Stanford University School of Medicine, Stanford, California 94305
}

\begin{abstract}
We recorded the responses of single neurons in extrastriate area MST while rhesus monkeys discriminated the direction of motion in a set of stochastic visual displays. By varying systematically the strength of a coherent motion signal within the visual display, we were able to measure simultaneously the monkeys' psychophysical thresholds for direction discrimination and the responses of single neurons to the same motion signals. Neuronal thresholds for reliably signaling the direction of motion in the visual display were calculated from the measured responses using a method based in signal detection theory. Neurons in MST were exquisitely sensitive to motion signals in the display, having thresholds for discriminating the direction of coherent motion that were, on average, equal to the psychophysical thresholds of the monkeys. For many MST neurons, the intensity of the response was correlated with the monkey's psychophysical judgements for repeated presentations of a given near-threshold stimulus; the monkey tended to choose the preferred direction of the neuron under study when that neuron responded more strongly to the stimulus. In both of these respects, MST neurons were indistinguishable from neurons in extrastriate area MT, a major source of afferent input to MST. In a second set of experiments, we found that both of these results held true in the face of pronounced manipulations of the visual stimulus. Severe reductions in stimulus size and speed, for example, compromised neuronal and psychophysical sensitivities by similar amounts so that the average neuronal and psychophysical thresholds remained approximately equal. In addition, the trial-to-trial covariation of neuronal response and perceptual decision was unaffected by our stimulus manipulations. Thus, MST neurons carry signals appropriate for supporting psychophysical performance on our task over an impressively wide range of stimulus configurations.
\end{abstract}

[Keywords: visual cortex, motion processing, MST, direction selectivity, direction discrimination, visual psychophysics]

\footnotetext{
Received Aug. 18, 1993; revised Dec. 2, 1993; accepted Dec. 21, 1993.

We are grateful to Judy Stein for excellent technical assistance during these experiments. K. H. Britten, C. D. Salzman, M. N. Shadlen, and E. Zohary made numerous helpful suggestions throughout the course of this work and preparation of the manuscript. This work was supported by the National Eye Institute (EY 05603) and by a postdoctoral fellowship to S.C. from the C.N.R.S. of France.

Correspondence should be addressed to Dr. William T. Newsome at the above address.

a + Present address: Centre de Recherche Cerveau et Cognition, CNRS-UPS.Faculte de Medecine Rangueil, 133 Route de Narbonne, 31062 Toulouse cedex., France. Copyright (C) 1994 Society for Neuroscience $0270-6474 / 94 / 144109-16 \$ 05.00 / 0$
}

Visual perception depends upon a cascade of neural activity that commences in the retina and reaches the highest levels of the cerebral cortex. The neural representation of the visual image is transformed at successive stages of this cascade so that behaviorally relevant information is extracted and made available to the rest of the system. In primates, for example, the first explicit representation of the direction of motion of objects in the visual world occurs within a functionally specialized "motion pathway" in the cerebral cortex (reviewed by Maunsell and Newsome, 1987; Zeki, 1991; Albright, 1993). The majority of neurons in this pathway are "directionally selective" in that they respond well to motion within a range of directions centered about a single "preferred" direction, but respond poorly or not at all to other directions of motion.

For the past several years we have used the cortical motion pathway as a model system for investigating the relationship between neural activity in the cerebral cortex and visual perception. Specifically, we have attempted to determine how neuronal signals within this pathway contribute to perceptual judgements in a simple task involving discrimination of the direction of motion in a set of stochastic visual displays. All of our experiments have been performed using alert macaque monkeys in which we measure and manipulate physiological and psychophysical events simultaneously. Having directed our efforts thus far toward the middle temporal visual area (MT) of the motion pathway, we have produced several lines of evidence that monkeys use directional information supplied by MT neurons to form perceptual judgements during our psychophysical task (Newsome and Paré, 1988; Newsome et al., 1989; Salzman et al., 1990, 1992; Britten et al., 1992; Murasugi et al., 1993). MT is but one area on the cortical motion pathway, however, and its role in mediating performance of our task cannot be understood in isolation from the neural system in which it is embedded. For example, the responses of MT neurons depend critically upon the nature of afferent input to MT, and the effects of MT neurons on perceptual behavior can only be expressed through their influence on "downstream" areas of the cerebral cortex and brainstem.

We have therefore begun to analyze activity in other areas of the motion pathway during performance of our direction discrimination task. In this report we describe the responses of directionally selective neurons in the medial superior temporal visual area (MST, or V5A), an area lying ventral and anterior to MT in the depths of the superior temporal sulcus. MST lies "downstream" to MT in the hierarchy of cortical visual areas since it is one of the major targets of "feedforward" outputs from MT (Maunsell and Van Essen, 1983; Ungerleider and Desimone, 1986; Felleman and Van Essen, 1991). Like MT, a large majority of neurons in MST are directionally selective, 
but MST receptive fields are substantially larger than those in MT (Van Essen et al., 1981; Desimone and Ungerleider, 1986; Tanaka et al., 1986). Both the increase in receptive field size and the extensive convergence of anatomical projections from MT to MST suggest that substantial pooling occurs as information is transmitted from M' to MST (Ungerleider and Desimone, 1986). We thus set out to determine how the responses of MST ncurons are related to psychophysical performance on our task, and to compare the sensitivity to motion signals of single neurons in MST and MT. As in our prior study of MT, the visual stimuli were tailored for each experiment to match as closely as possible the receptive field properties of the neuron under study.

We have found that the responses of MST neurons to our stochastic visual stimuli are, in most respects, indistinguishable from those of MT neurons. Neuronal thresholds for signaling the direction of motion in our displays are similar in the two areas and are thus similar, on average, to psychophysical thresholds generated by the monkeys on the same sets of trials. In addition, neurons in MST as well as MT exhibit a trial-to-trial covariation between the intensity of single-neuron responses and the monkey's decision regarding the direction of motion for near-threshold stimuli. This covariation suggests that neurons in MST and MT actually influence the monkey's decision on individual trials. Thus, simple motion signals like those used during performance on our task appear to be transmitted faithfully from MT through the complex circuitry of MST to higher cortical centers that implement perceptual decisions. Such fidelity may require a rather precise balance between sensitivity gains resulting from pooling of MT afferents and sensitivity losses due to noise sources intrinsic to MST.

In a second set of experiments in MST, we attempted to destroy the similarity of neuronal and psychophysical thresholds by using motion stimuli substantially different from those preferred by MST neurons. In the extreme case, we reduced the size of the visual stimulus aperture to $3^{\circ}$ in diameter and reduced the speed of the random dot motion signal to 0.4 degree $/ \mathrm{sec}$, values that seemed roughly appropriate for neurons in lower areas of the motion pathway such as V1, V2, and V3. These stimulus manipulations reduced the responsiveness of most MST neurons and thus elevated their thresholds for signaling the direction of motion. However, psychophysical thresholds were elevated by roughly the same amount so that, on average, the identity between neuronal and psychophysical performance remained intact. Similarly, the trial-to-trial covariation between neuronal response and perceptual decision was unaffected by these stimulus manipulations. MST ncurons may therefore provide motion signals that support performance on our task over a broad range of speeds and aperture sizes.

A brief report of these experiments has appeared previously (Celebrini and Newsome, 1992).

\section{Materials and Methods}

\section{Subjects, surgery, and daily routine}

The experiments were performed on two adult rhesus monkeys weighing between 7 and $9 \mathrm{~kg}$ (Macaca mulatta, one male and one female). Prior to the experiments, each monkey was surgically implanted with a device for stabilizing head position (Evarts, 1968), a scleral search coil for measuring eye movements (Judge et al., 1980), and a recording cylinder that allowed microelectrode access to visual cortex within the occipital lobe. All surgical procedures were performed under aseptic conditions with halothane anesthesia. After recovery from surgery, each animal engaged in daily training or experimental sessions of $2-6 \mathrm{hr}$ duration.
Behavioral control was accomplished by operant conditioning techniques using fluids as a positive reward; fluid intake was therefore restricted during periods of training or electrophysiological recording. The animals were maintained in accordance with the guidelines set by the U.S. Department of Health and Human Services (NIH) Guide for the Care and Use of Laboratory Animals.

\section{Visual stimuli}

The visual stimuli employed in this study were a set of dynamic random dot stimuli in which a unidirectional motion signal was interspersed amongst random motion noise. This stimulus set has been described extensively in previous publications (e.g., Britten et al., 1992), and we simply summarize its essential features here. The visual display consists of a stream of dots plotted in rapid succession at specified locations on a CRT screen. Although the dots are plotted sequentially, persistence within the visual system is such that many dots appeared to be present simultaneously. The strength of the motion signal can be varied from trial to trial by varying the proportion of dots appearing to move in a particular direction. In practice, this proportion is established by specifying the probability that a vanishing dot will reappear with a fixed displacement in space and time, and we refer to this proportion as the "correlation" of the visual stimulus. In a $0 \%$ correlation stimulus, for example, all dots are plotted at random locations and there is no net motion signal in any single direction. In a $100 \%$ correlation stimulus, on the other hand, all dots are replottcd with a constant displacement in space and time and every dot appears to move smoothly in the specified direction. The motion signal can assume any strength between these two extreme values, allowing precise control of the difficulty of the psychophysical discrimination.

The temporal interval between successive plottings of "signal" dots was always $45 \mathrm{msec}$; the magnitude of the spatial displacement was varied so as to match the speed of the motion signal (spatial interval divided by temporal interval) to the preferred speed of the neuron under study. The stimuli were presented on a large CRT screen placed $38 \mathrm{~cm}$, or occasionally $57 \mathrm{~cm}$, in front of the monkey and were generated by a PDP11/73 computer.

\section{Electrophysiological recording and behavioral paradigms}

Electrophysiological recordings were made with tungsten microelectrodes inserted into the cortex through a transdural guide tube (electrode impedance, $0.5-2 \mathrm{M} \Omega$ at $1 \mathrm{kHz}$; Frederick Haer Inc., Microprobe Inc.). The guide tube was held in place by a plastic grid positioned inside the recording cylinder (Crist et al., 1988). This grid provided a stable coordinate system for positioning guide tubes; we recorded through any particular guide tube for several consecutive days. The signal from the microelectrode was amplified and action potentials were isolated from single neurons using a time-amplitude window discriminator (Bak Electronics). The time of occurrence of each action potential was measured with $1 \mathrm{mscc}$ resolution and stored on a computer disk for later analysis. Unit activity was monitored on line with raster displays and an audio monitor. The monkey's eye movements were measured throughout the experiment using the scleral search coil technique. If the monkey broke fixation during the stimulus presentation period, we aborted the trial and discarded the data.

Fixation task. During this task, the monkey was required simply to maintain its eye position within an electronically defined window around the fixation point for a specified interval of time, usually $2-4$ sec. The monkey received a liquid reward for each successfully completed trial. In most experiments, the window permitted eye movements up to $1.5^{\circ}$ away from the fixation point, but in practice, the monkeys usually maintained their eye position within $0.5^{\circ}$ of the fixation point.

The monkeys performed the fixation task during the initial search for a single unit, during qualitative mapping of the receptive field, and during quantitative measurement of direction and speed tuning curves. To obtain a direction tuning curve, a $100 \%$ correlated dot pattern was presented in eight different directions of motion that were equally spaced at $45^{\circ}$ intervals. The different directions were presented in a random sequence until six to eight repetitions were obtained for each direction. The optimal speed was estimated qualitatively for all cells, but quantitative speed tuning curves were measured for a minority of cells. For these experiments, the $100 \%$ correlated dot pattern was presented in both the preferred and null directions at seven different speeds, ranging from $0.4 \mathrm{degree} / \mathrm{sec}$ to $28.4 \mathrm{degrees} / \mathrm{sec}$. Again, the various stimulus 
conditions were presented in random order until six repetitions were obtained for cach condition.

Discrimination task. The bulk of our data were obtained while the monkey performed a two-alternative, forced-choice direction discrimination task (see Britten et al., 1992, for a detailed description). Collection of these data followed receptive field mapping and measurement of tuning curves. On each trial, a random dot motion stimulus was presented for $2 \mathrm{sec}$ within a circular aperture adjusted to match as closely as possible the location and dimensions of the neuron's receptive field. The direction of the correlated motion signal was varied randomly from trial to trial between the preferred and null direction of the neuron under study, and the monkey's task was to discriminate correctly the direction of motion. The strength of the motion signal was also varied systematically among several correlation levels chosen to span psychophysical threshold. A minimum of 15 repetitions was obtained for each stimulus condition (i.c., cach combination of direction and correlation lcvel), and all conditions were presented in a randomly ordered sequence. During each presentation of a visual stimulus, the monkey was required to maintain its eye position on the fixation point so that the random dot motion pattern was positioned at a consistent location within the neuron's receptive field. In this manner we created a psychophysical situation in which the monkey's decision was likely to depend on directional signals like those provided by the recorded neuron.

A single trial began with appearance of the fixation point. After the monkey achieved fixation, the visual stimulus was presented as described above. At the end of the $2 \mathrm{sec}$ display interval, the random dot pattern and the fixation point disappeared and two light-emitting diodes (LEDs) appeared, each corresponding to one of the two possible directions of correlated motion. To indicate its decision regarding the direction of motion, the monkcy made a saccadic cyc movement to the appropriate LED. The monkey received a liquid reward for correct decisions; a brief "time-out" period followed incorrect decisions. On $0 \%$ correlation trials, the monkey was rewarded randomly with a probability of 0.5 since there was no correct answer on these trials. If the monkey broke fixation prior to the end of the stimulus presentation interval, the trial was aborted and the data discarded.

\section{Mapping the receptive field and positioning the stimulus aperture}

Receptive fields were mapped on a tangent screen positioned directly in front of the monkey; the dimensions of the tangent screen were $110^{\circ}$ $\times 110^{\circ}$ (corrected for tangent error). A large bar of light was typically used to establish the boundaries of the receptive field on the tangent screen. For the few units that were unresponsive to bars, we used large random dot patterns to locate responsive regions of the visual field, but we did not attempt to establish the boundaries of these receptive fields with any precision. In addition, we did not attempt to locate any receptive field boundaries that fell beyond the edges of the tangent screen.

After mapping the receptive field, we attempted to match the location and size of the visual stimulus aperture to the location and size of the receptive field. This was frequently impossible, however, because of the very large size of MST receptive fields. Using a large screen HP 1321 oscilloscope and a viewing distance of $38 \mathrm{~cm}$, our largest achievable stimulus aperture was $30^{\circ}$ in diameter. Since the mean diameter of our MST receptive fields was approximately $55^{\circ}$, the visual stimulus aperture was usually smaller than the visual receptive field. When this occurred, we attempted to center the stimulus aperture on the most responsive portion of the receptive field. On rare occasions, the most responsive portion of the receptive field was too eccentric for the monkey to perform well psychophysically, in which case we placed the stimulus aperture on a less responsive region of the receptive field nearer to the center of gaze. When the neuron responded equally well throughout the receptive field, we positioned the stimulus within the portion of the receptive field closest to the center of gaze so as to facilitate the monkey's psychophysical performance.

\section{Analysis of psychophysical data}

The psychophysical data obtained during each experiment were compiled into psychometric functions depicting the proportion of correct decisions as a function of the strength of the motion signal (in percentage correlation). We used a maximum likelihood method (Watson, 1979) to fit these data with sigmoidal functions of the form

$$
p=\delta-(\delta-0.5) \exp \left[-(c / \alpha)^{\prime}\right]
$$

where $p$ is the probability of correct decision, $c$ is the stimulus correlation, $\alpha$ is the level of correlation supporting threshold performance ( $82 \%$ correct responses), $\beta$ is the slope of the function, and $\delta$ is the asymptotic performance level obtained for strong motion signals (expressed as proportion of correct decisions). The threshold parameter, $\alpha$, and the slope parameter, $\beta$, provide a succinct description of the psychophysical data and will be used extensively for this purpose throughout this report.

Equation 1, derived from the integral of a Weibull distribution (Quick, 1974), fitted our psychophysical data well. Of the 120 psychometric functions in our data set, 112 were well fit when the asymptotic performance variable, $\delta$, was constrained to be unity ( $\chi^{2}$ test, $p>0.05$ ), and six of the eight remaining functions were well fit by allowing $\delta$ to be a free parameter. The nonunity asymptote in the latter six experiments reflects the fact that the monkey made a small number of errors at the highest correlation levels.

\section{Analysis of physiological data}

We analyzed our physiological data in a manner that permitted a direct comparison of neuronal sensitivity to psychophysical sensitivity (see Britten et al., 1992, for a detailed description). For each neuron, we first compiled for each motion correlation level a distribution of responses to preferred direction motion and a separate distribution of responses to null direction motion. A "response" was considered to be the total number of spikes generated by the neuron during a given $2 \mathrm{sec}$ stimulus presentation. For very strong motion signals (high correlation levels), these preferred and null response distributions did not overlap, and the direction of motion in the stimulus was therefore signaled unambiguously by this neuron. For weak motion signals, however, the preferred and null response distributions overlapped considerably, and the neuron thus provided very poor information about the direction of stimulus motion. Intermediate levels of overlap between the preferred and null response distributions would yield intermediate levels of discriminative ability.

Following these intuitions, we used a method based on signal detection theory (Green and Swets, 1966) to compute the performance level expected of an ideal observer who based judgements of motion direction upon the measured neuronal responses. To perform this calculation, we assumed that the observer's decisions depended upon a comparison on each trial of the responses of two neurons-the one under study and an identical neuron that preferred the opposite direction of motion (the "antineuron"). Using this assumption, the preferred and null response distributions of the neuron under study also represent the responses of the antineuron, with a simple reversal of the preferred and null directions. On any given trial, the ideal observer would make a decision concerning the direction of motion by comparing a random draw from the preferred direction response distribution of one neuron to a random draw from the null direction response distribution (at the same stimulus correlation level) of its antineuron. At each correlation level, therefore, the discriminative ability of an MT neuron could be characterized as the probability that a randomly selected response from the preferred direction distribution was larger than a randomly selected response from the null direction distribution. (Note that this decision rule is particularly advantageous in that it requires no independent neural representation of a "noise" level that would influence performance; decisions are based simply upon a comparison of two responses that are present simultaneously within the cortex during each stimulus presentation.)

The performance expected of the ideal observer was calculated for each correlation level by measuring the area under an ROC curve (receiver operating characteristic) constructed from the preferred and null response distributions (Green and Swets, 1966; Britten et al., 1992). For each neuron, the resulting probabilities were compiled into a "neurometric function" that plotted expected performance (expressed in percentage correct decisions) as a function of correlation level. A sigmoidal curve was fitted to the data using Equation 1, and threshold and slope parameters were extracted from the fitted curve. These parameters capture the sensitivity of an individual neuron to motion signals in our display in a manner that can be compared directly to the psychophysical sensitivity of the monkey measured on the same set of trials. Equation 1 described our neurometric data well; the fit could be rejected for only a single neuron out of 120 in our sample ( $\chi^{2}$ test, $p<0.05$ ).

Signal detection theory has been usefully applied to the analysis of neurophysiological data like ours in several prior investigations (e.g., Barlow et al., 1971; Cohn et al., 1971; Tolhurst et al., 1983; Bradley et 


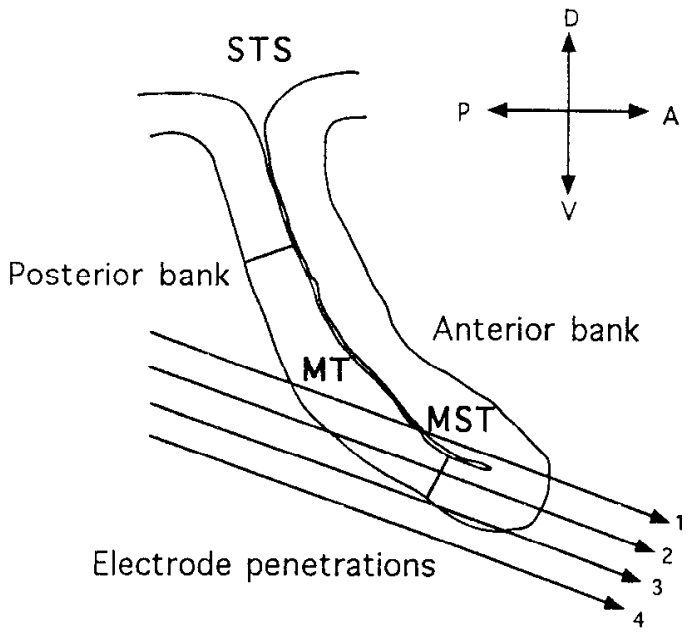

Figure 1. Electrode trajectories through the superior temporal sulcus (STS) commonly encountered in our experiments. The section outline shows a typical STS profile in brains sectioned parasagittally. The middle temporal area $(M T)$, whose boundaries can be identified on myelin stained sections, lies on the posterior bank of the sulcus; the medial superior temporal area (MST) occupies the fundus and anterior bank of the sulcus. Electrode penetrations approach the STS from posterior to anterior at a $20^{\circ}$ angle with respect to the horizontal plane.

al., 1987; Hawken and Parker, 1990; Vogels and Orban, 1990; Zohary et al., 1990).

\section{Quantitative comparison of psychometric and neurometric functions}

For each experiment, the preceding analyses yielded a pair of sigmoidal functions that described psychophysical and neuronal sensitivity to the motion signals in the set of visual stimuli. To compare quantitatively the psychophysical and neuronal sensitivity observed in a single experiment, we tested for homogeneity of the two data sets using a statistical approach introduced for this purpose by Britten et al. (1992). In essence, this approach tests the null hypothesis that the neural and psychophysical data are described no better by two separate Quick functions than by a single, common function. Using maximum likelihood methodology, we fitted separate Quick functions to the psychometric and neurometric data, and we fitted the best single Quick function to the two data sets considered jointly. The log likelihoods obtained from these two conditions are transformed by

$$
\lambda=-2 \ln (L \text { (data } \mid \text { single curve }) / L \text { (data } \mid \text { independent curves)), }
$$

so that $\lambda$ is distributed as $\chi^{2}$ with 2 degrees of freedom (see Hoel et al., $\mathrm{p} 83 \mathrm{ff}$ ). If $\lambda$ does not exceed the criterion value (for $p=0.05$ ), we conclude that a single function fits the two data sets no worse than two separate functions.

For experiments in which the neurometric and psychometric data were demonstrably different, we wished to determine whether the disparity arose from a difference in threshold $(\alpha)$, slope $(\beta)$, or both. We again tested the null hypothesis that one curve described the neural and psychophysical data as well as two curves, elaborating the procedure to compare three conditions: (1) assuming a common threshold $(\alpha)$ for the neural and psychophysical data, (2) assuming a common slope $(\beta)$ for the two data sets, and (3) allowing both the threshold and slope parameters to vary freely. If two curves with slopes constrained to be equal described the data significantly better than a single curve, we inferred that the difference between neural and psychophysical data arose from a disparity in thresholds. Conversely, if the data were best described by two curves with thresholds constrained to be equal, we concluded that the difference arose from a disparity in slopes.

\section{Identification of MST}

Both of the monkeys used in this study are still alive and participating in related experiments. Since histological confirmation of our recording sites is not yet possible, we will describe precisely how we assigned

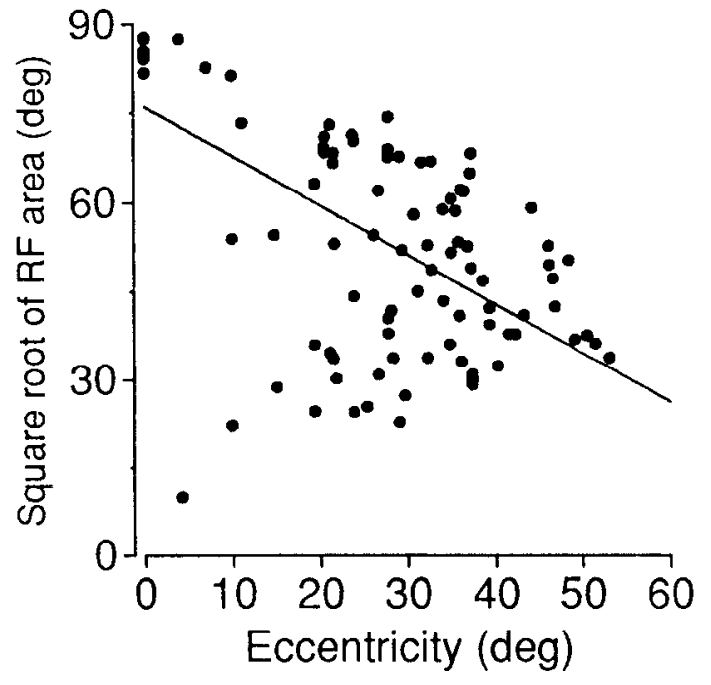

Figure 2. Square root of receptive field $(R F)$ area as a function of the eccentricity of the RF center. Data are shown for 107 MST neurons; we were unable to obtain an accurate receptive field map for 13 neurons that failed to respond to bars or spots of light.

neurons to area MST and the reasons for our confidence in these assignments.

In all of our experiments, we employed a posterior approach to the superior temporal sulcus. The electrode traveled in a parasagittal plane, tilted approximately $20^{\circ}$ with respect to the horizontal plane. Thus, the guide tube and electrode typically passed through striatc cortcx and the lunate sulcus before entering the posterior bank of the superior temporal sulcus (STS).

Figure 1 schematizes several electrode trajectories through the STS that were commonly encountered in a prior study using the same cylinder placement and approach angle. For well-targeted passes like penetration 1, the middle temporal area (MT) was the first visual area encountered upon arrival of the electrode at the posterior bank of the STS. MT, which had been previously mapped in both of our animals, is recognizable from a combination of features including the characteristic direction selectivity and receptive field size of its neurons, and its topographic map of the contralateral visual hemifield. After leaving MT, this electrode track crossed the lumen of the STS and entered MST on the anterior bank of the sulcus. The lumen of the sulcus was almost always recognizable by the absence of ncuronal activity as the clcctrodc crossed from one bank to the other. On some passes, such as penetration 2 in Figure 1, the electrode traveled tangentially along the sloped floor of the STS and therefore moved from MT into MST without crossing the lumen of the sulcus. Finally, electrode passes occasionally took the trajectory of penetration 3, intersecting the STS near its fundus and therefore entering MST directly without prior passage through MT (Van Essen et al., 1981). An encounter with the fundus could be confirmed simply by running the next penetration slightly more ventrally and ascertaining that the electrode missed the STS altogether (as in penetration 4). Using our angle of approach, we never reenter MT after arriving in MST because the entire body of MST lies anterior and/or ventral to MT. As described below, we rely heavily on this fact in assigning recording sites to MST.

Neurons in MST, like those in MT, arc typically dircctionally selective, but the average receptive field size of MST neurons is substantially larger than that in MT (Van Essen et al., 1981; Desimone and Ungerleider, 1986). While the increase in receptive field size is very helpful in distinguishing MST from MT, it cannot be used uncritically since there is substantial overlap in receptive field sizes observed in the two areas (Tanaka et al., 1986; Komatsu and Wurtz, 1988). When mapping cortex in the STS, therefore, it is not uncommon to encounter a region of uncertainty in which receptive field size alone does not permit unambiguous assignment of neurons to MST or MT. This occurs most frequently when crossing from MT to MST along the floor of the STS or when encountering MST in isolation on penetrations near the fundus. In these cases, we adopted a conservative procedure and refused to assign neurons to MST until we obtained unambiguous evidence that 


\begin{tabular}{lllll}
\hline Table 1. & Detailed description of data set & & \\
& & & Cells & $\begin{array}{l}\text { Cells } \\
\text { included }\end{array}$ \\
Monkey & Guide tubes & Penetrations & screened & 70 \\
K & 12 & 95 & 206 & 50 \\
E & 13 & 121 & 141 & 5 \\
\hline
\end{tabular}

the electrode was indeed in MST. This evidence could take any of three forms: (1) receptive fields whose sizes were clearly larger than those reported previously for MT (on the order of a visual quadrant), (2) very large receptive fields that included the fovea (the largest MT receptive fields are in the representation of the periphery and do not approach the fovea), or (3) inclusion of a substantial portion of the ipsilateral hemifield within the receptive field (MT receptive fields can spread across the midline in to the ipsilateral hemifield, but rarely by more than 20. Albright and Desimone, 1987; Maunsell and Van Essen, 1987). After identifying a cell or cells as belonging unambiguously to MST, all successive cells on the penetration were assigned to MST, regardless of receptive field size, since our angle of approach precluded reentry into MT (see above).

Several groups have presented evidence that MST, as originally defined by Van Essen and colleagues, should be subdivided into distinct functional areas (Desimone and Ungerleider, 1986; Saito et al., 1986; Newsome et al., 1988; Tanaka and Saito, 1989). We directed the bulk of our penetrations toward the dorsomedial subdivision (MSTd) since neurons in this region respond particularly well to extended dot patterns like those employed in our study. We are sure, however, that a few of our penetrations encountered the ventrolateral subdivision (MST1), and these penetrations account for some of the smaller receptive fields in our sample.

Figure 2 illustrates the size-eccentricity relationship of 107 MST receptive fields. Sizes are represented by the square root of receptive field area, and eccentricity is the visual angle between the fovea and the center of the receptive field. Both the receptive field sizes and eccentricities have been corrected for tangent error, which can be substantial for the large, eccentric receptive fields frequently encountered in this study. We note, however, that the size and eccentricity of our largest receptive fields were underestimated because we did not map beyond the limits of our tangent screen. The scatterplot in Figure 2 shows a significant negative relationship between the receptive field size and eccentricity $(r=0.60, p<0.0001)$, but the negative relationship is driven mostly hy a few neurons with extremely large receptive fields covering both hemifields and therefore centered on the fovea. The sizeeccentricity data illustrated in Figure 2 are very similar to those reported by Tanaka et al. (1986) and by Tanaka and Saito (1989), with the one exception that the prior investigators reported more small receptive fields than we observed. This discrepancy probably results from our very conservative physiological criteria for classifying cells as belonging to MST (see above). Desimone and Ungerleider (1986), however, reported a positive relationship between receptive field size and eccentricity in MSI, parallel to that observed in MT. The sole difference between Desimone and Ungerleider's data and our own is that these investigators did not observe large receptive fields centered on the fovea; the two data sets are very similar otherwise. The overall agreement of the data in Figure 2 with those reported by other investigators reinforces our conclusion that our conservative physiological criteria enabled us to assign neurons to MST accurately.

\section{Results}

Our results are based on recordings from 120 MST neurons in two monkeys; Table 1 provides a morc detailed description of the data set. We first describe the basic responses of MST neurons to our stochastic motion stimuli, including their direction selectivity and response variance. Because both of these properties influence neuronal thresholds for signaling direction of motion, these data are relevant to the subsequent comparison of neuronal and psychophysical sensitivity.

We admitted neurons to the data set on the basis of two criteria: (1) responsiveness to our stochastic stimuli, and (2)

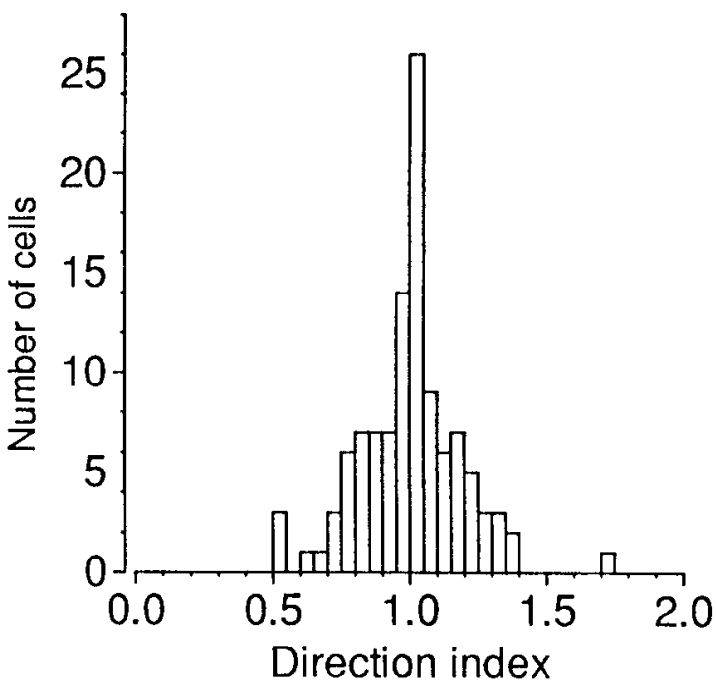

Figure 3. A frequency histogram of direction indices from 111 MST neurons for which direction tuning data were obtained. We used a conventional index of directionality, $1-N / P$, where $P$ is the response of the neuron to preferred direction motion and $N$ is the response to the opposite, or null, direction. Maintained activity was subtracted from the responses before the direction index was calculated. Indices near unity indicate strong direction selectivity, while indices near zero represent poor direction selectivity. Direction indices greater than unity result from inhibition to null direction motion.

reliable direction selectivity in the sense that all responses to the strongest preferred direction stimulus (100\% correlation) were larger than all responses to the strongest null direction stimulus. These criteria resulted in well-defined neurometric functions since performance (of an ideal observer) based on such responses asymptotes at $100 \%$ correct for the strongest motion signal (see Materials and Methods). Note that these criteria admitted both directionally biased and directionally selective cells to the data base as long as the preferred and null direction response distributions were nonoverlapping. Figure 3 illustrates the distribution of direction indices computed for the 120 neurons in our sample. This direction index ranges from a value of 0 for completely nondirectional cells to a value of unity for cells that respond only to preferred direction motion (see formula in the legend to Fig. 3). The index may assume values greater than unity if the neuron is actually inhibited by null direction motion. The mean of the distribution in Figure 3 is $1.0( \pm 0.18)$, showing that our MST neurons were, on average, highly directional. This distribution, of course, is not descriptive of MST as a whole since we excluded neurons that failed to meet the criteria described above.

Figure 4 illustrates the relationship between the mean responses of our MST cells and the corresponding response variance. Since the response of an MST cell usually varies systematically with the direction and strength of the motion signal, the data points in Figure 4 represent each individual motion condition tested for each cell. The data are well fitted by a straight line on the log-log plot in Figure 4, indicating that mean and variance are related according to a power function of the form

$$
\text { variance }-a(\text { mean })^{b} \text {, }
$$

where $b$ is the slope of the relationship and $a$ is the intercept. A slope of 1.12 and an intercept of 1.09 , illustrated by the regression line in Figure 4, provide the best fit to our data. These 


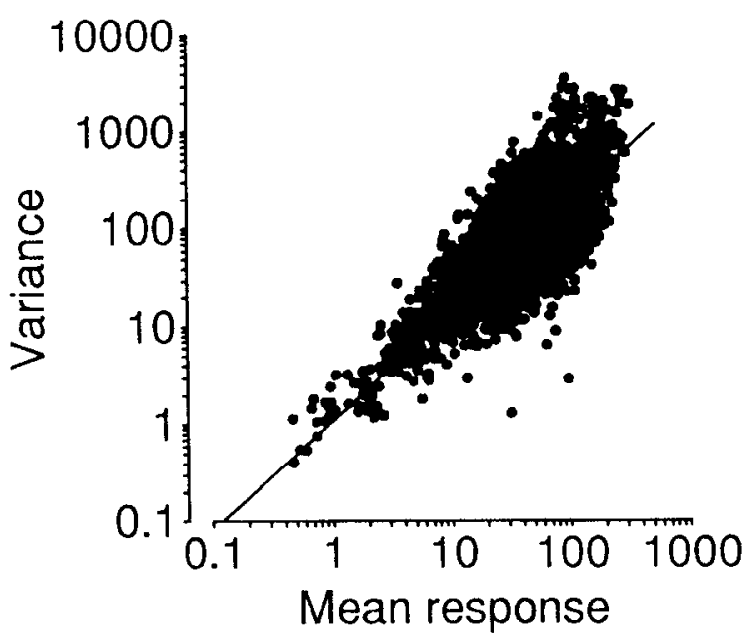

Figure 4. Relationship between mean response level and response variance for $120 \mathrm{MST}$ neurons. The variance and mean response were calculated and plotted for each stimulus condition tested for each cell. The best-fitting regression is indicated by the solid line.

values are similar to those reported in previous studies in other laboratories, showing that MST neurons conform to the same type of variance-to-mean law as neurons in other visual areas (Dean, 1981; Tolhurst et al., 1983; Vogels et al., 1989; Snowden et al., 1992; Britten et al., 1993).

\section{Comparison of psychometric and neurometric functions}

Figure 5 illustrates the neurometric and psychometric data obtained in six representative experiments. For each experiment, the open symbols and dashed lines depict the monkey's psychophysical performance, while the solid symbols and solid lines represent neuronal performance measured on the same set of trials (see Materials and Methods). In Figure 5, $A$ and $B$ show the results of two experiments in which the psychometric and neurometric data were essentially indistinguishable, the two functions having similar thresholds as well as similar slopes. In Figure $5 A$, for example, the psychophysical and neuronal thresholds were $8 \%$ and $8.4 \%$ correlated dots, respectively, and the unitless slope parameters for the two functions were 1.15 and 1.16 , respectively. Psychophysical and neuronal thresholds were somewhat lower in the experiment of Figure $5 B(4 \%$ and $5.1 \%$ correlated dots, respectively) and the slopes of the two functions were steeper (slope parameters $=1.6$ and 1.7 , respectively). Figure 5, $C$ and $D$, illustrates experiments in which the MST neuron was significantly more sensitive than the monkey. Though the slopes of the two functions were similar in these experiments, psychophysical threshold was roughly twice as high as neuronal threshold (precise values for thresholds and slopes are given in the legend). Finally, Figure $5, E$ and $F$, depicts experiments in which the neuron was significantly worse than the monkey's psychophysical performance.

Using a statistical approach outlined in Materials and Methods, we tested each pair of neurometric and psychometric functions to determine whether the two data sets differed significantly. The neurometric and psychometric data were indistinguishable for 70 of our 120 experiments $(58.3 \%)$, including the experiments of Figure 5, $A$ and $B$. For the experiments in which the neurometric and psychometric functions differed, we performed a more extensive analysis to determine whether the disparity arose from a difference in threshold $(\alpha)$
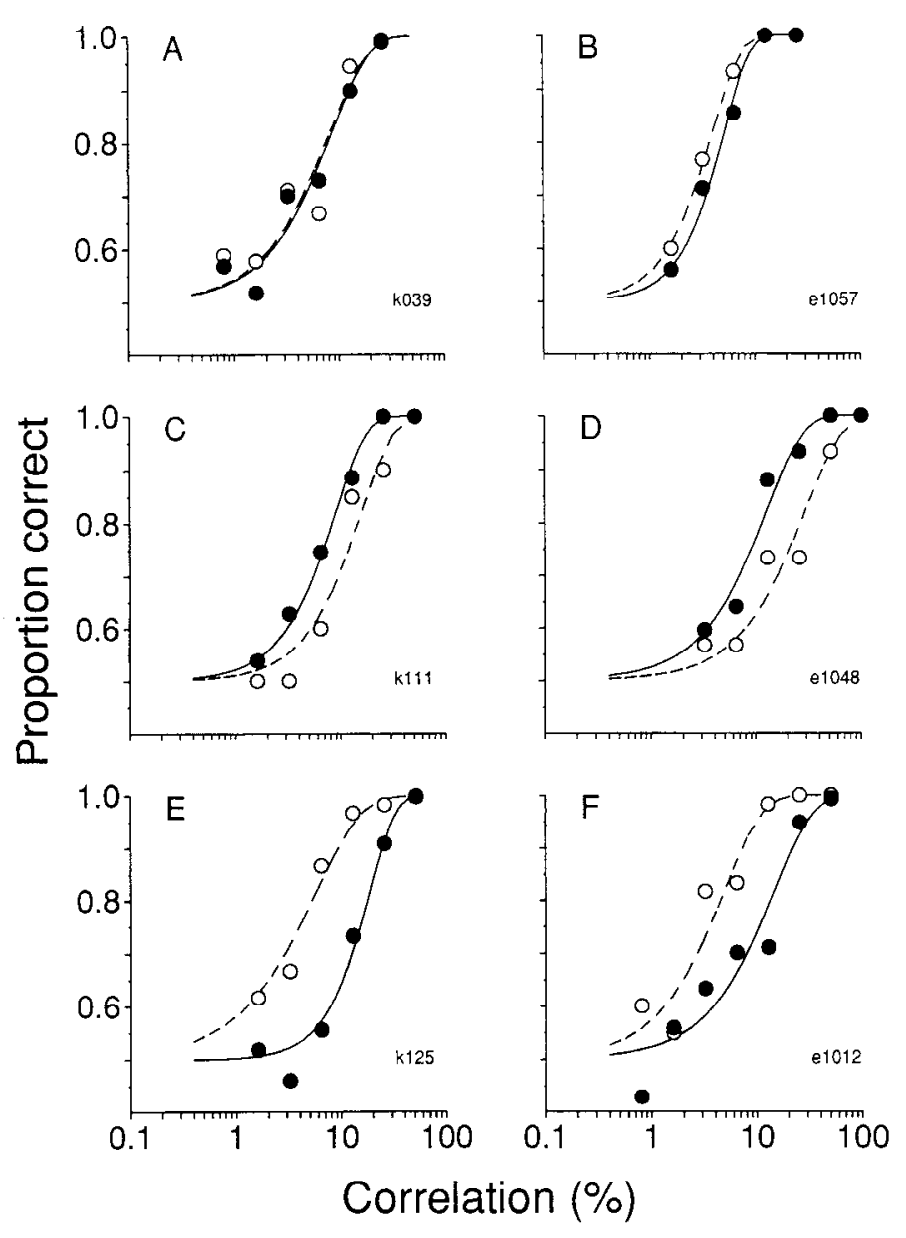

Figure 5. Psychometric and neurometric functions obtained in six MST experiments. The open symbols and broken lines describe psychometric data; the solid symbols and solid lines describe neurometric data. $A$, An experiment in which the psychometric and neurometric functions were statistically indistinguishable. Psychometric $\alpha=8 \%$ correlation, $\beta=1.15$; neurometric $\alpha=8.4 \%$ correlation, $\beta=1.16 . B, \mathrm{~A}$ second experiment in which psychometric and neurometric functions were indistinguishable. Psychometric $\alpha=4 \%$ correlation, $\beta=1.6$; neurometric $\alpha=5.1 \%$ correlation, $\beta=1.7$. $C$, An experiment in which the neural threshold was lower than the psychophysical threshold. Psychometric $\alpha=15.5 \%$ correlation, $\beta=1.38$; neurometric $\alpha=8.8 \%$ correlation, $\beta=1.41 . D$, A second experiment in which the neuron was more sensitive. Psychometric $\alpha=28.4 \%$ correlation, $\beta=1.19$; neurometric $\alpha=12.3 \%$ correlation, $\beta=1.19$. $E$, An experiment in which the psychophysical threshold was lower than the neuronal threshold. Psychometric $\alpha=5.7 \%$ correlation, $\beta=1.0$; neurometric $\alpha=18.5 \%$ correlation, $\beta=1.7$. $F$, A second experiment in which the psychophysical observer was more sensitive. Psychophysical $\alpha=4.8 \%$ correlation, $\beta=1.16$; neurometric $\alpha=14.5 \%$ correlation, $\beta=1.13$.

or slope $(\beta)$, as described in Materials and Methods. In most cases $(74 \%)$, a difference in threshold accounted for disparity between neural and psychophysical data, while a difference in slope accounted for the disparity in a small minority of cases $(6 \%)$. For the remaining $20 \%$, the disparity between neural and psychophysical data could not be attributed to either threshold or slope differences alone.

The overall similarity of neuronal and psychophysical thresholds is evident in Figure 6, which shows a frequency histogram of the ratio of these two measures for the 120 experiments in our data sample. Ratios less than unity indicate experiments in which neuronal thresholds were lower than psychophysical 


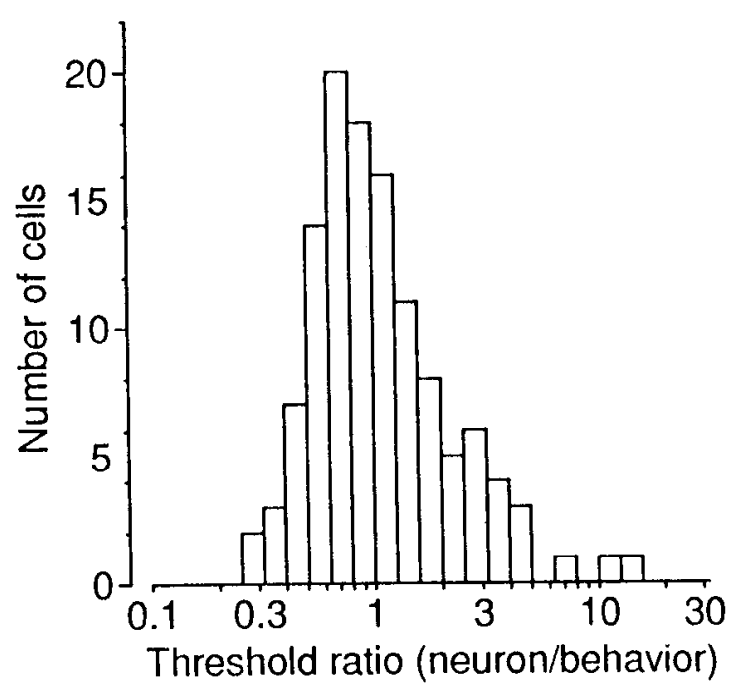

Figure 6. Relative sensitivities of single MST neurons and animal observers. The frequency histogram shows the ratio of neuronal threshold $(\alpha)$ to psychophysical threshold $(\alpha)$ for the 120 experiments in our sample. Ratios greater than unity indicate that the psychophysical observer was more sensitive than the neuron; ratios less than unity indicate that the neuron was more sensitive.

thresholds. On the other hand, ratios greater than unity represent experiments in which neuronal performance was worse than psychophysical performance. The range of the distribution in Figure 6 is large, but the geometric mean is almost exactly unity (1.06), indicating that average neuronal threshold was rather precisely equivalent to average psychophysical threshold. The combined distribution in Figure 6 obscures small but significant differences between the two animals. Considered individually, the mean threshold ratio was 0.87 for monkey $E$ and 1.22 for monkey $\mathrm{K}(t$ test, $p<0.01)$.

The shapes of the neurometric and psychometric functions, represented by the slope parameter, $\beta$, of the Weibull fits, were also quite similar on average. Figure 7 shows a frequency histogram of the ratio of the slopes of the neurometric and psychometric functions. The distribution of slope ratios is actually narrower than the distribution of threshold ratios, but the geometric mean was again near unity (1.13) and agreed closely for the two monkeys ( 1.16 for monkey $\mathrm{K} ; 1.10$ for monkey $\mathrm{E}$ ). The mean slope was 1.55 for the neurometric data and 1.37 for the psychometric data.

While Figures 6 and 7 demonstrate a close correspondence of average neuronal and psychophysical performance, they do not indicate whether variations of neuronal and psychophysical threshold about their means are correlated on an experimentby-experiment basis. Figure 8 addresses this question by means of a scatterplot of neuronal versus psychophysical threshold for all 120 MST experiments in our sample. Psychophysical thresholds varied widely across the data set since several stimulus parameters were changed for each experiment in order to match the physiological properties of the neuron under study. Neuronal thresholds varied even more broadly because of differences in the degree of direction selectivity among the neurons in our sample.

Figure 8 reveals a positive relationship between neuronal and psychophysical threshold $(r=0.61, p<0.0001)$, but a regression analysis demonstrated that this relationship can be attributed largely to an independent variable, speed of the motion signal,

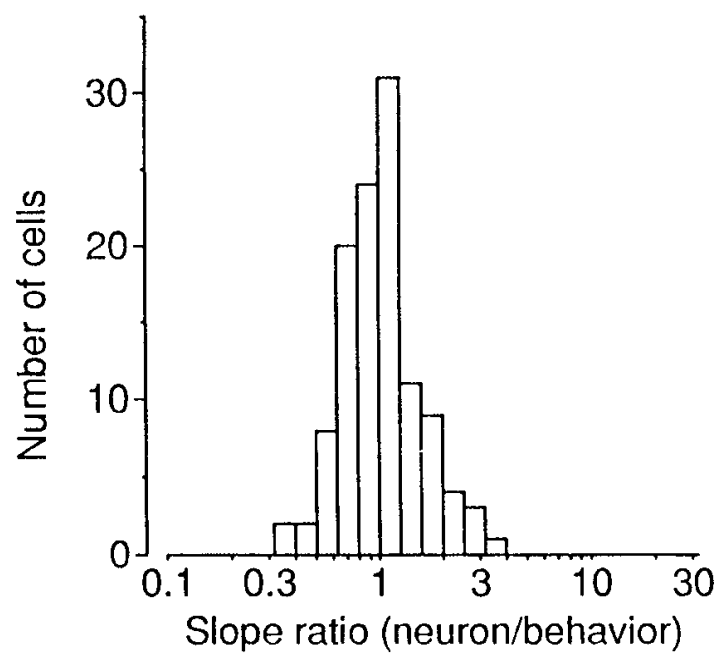

Figure 7. Relative slopes of neurometric and psychometric functions. The frequency histogram shows the ratio of the slope of the neurometric function $(\beta)$ to the slope of the psychometric function $(\beta)$ for the 120 experiments in our sample. Ratios less than unity indicate that the psychometric function was steeper than the neurometric function; ratios greater than unity indicate the converse.

that influences both psychophysical and neuronal thresholds. Stimulus speed alone accounted for $35 \%$ of the variance in psychophysical threshold, with thresholds increasing for experiments in which higher speeds were employed (single regression, $p<0.0001$ ). Inclusion of neuronal threshold as a second independent variable accounted for only $7 \%$ of additional variance in psychophysical threshold (multiple regression, $p<0.001$ ). A separate analysis confirmed that motion speed had a weakly significant, positive effect on neuronal threshold as well (single regression, $p=0.052$ ).

The observation that motion speed affects psychophysical thresholds on our task is not novel. We have frequently observed that thresholds for monkey and human observers are lowest for speeds extending roughly from 2 to 10 degrees/sec, but rise for

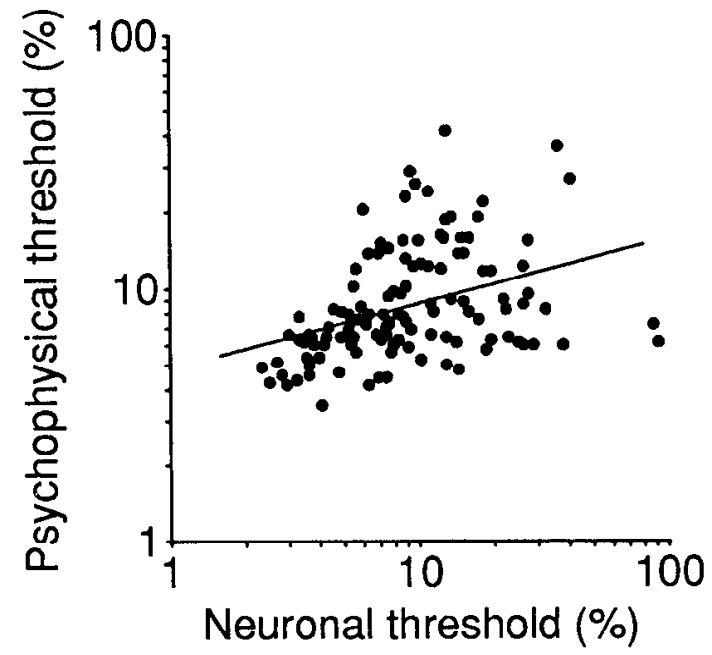

Figure 8. A scatterplot of neuronal threshold against psychophysical threshold for the 120 neurons in our sample. The solid line is the straight line that best fits the data (linear regression, $r=0.37, p \leq 0.0001$ ). 
Preferred decision Opposite dęcision
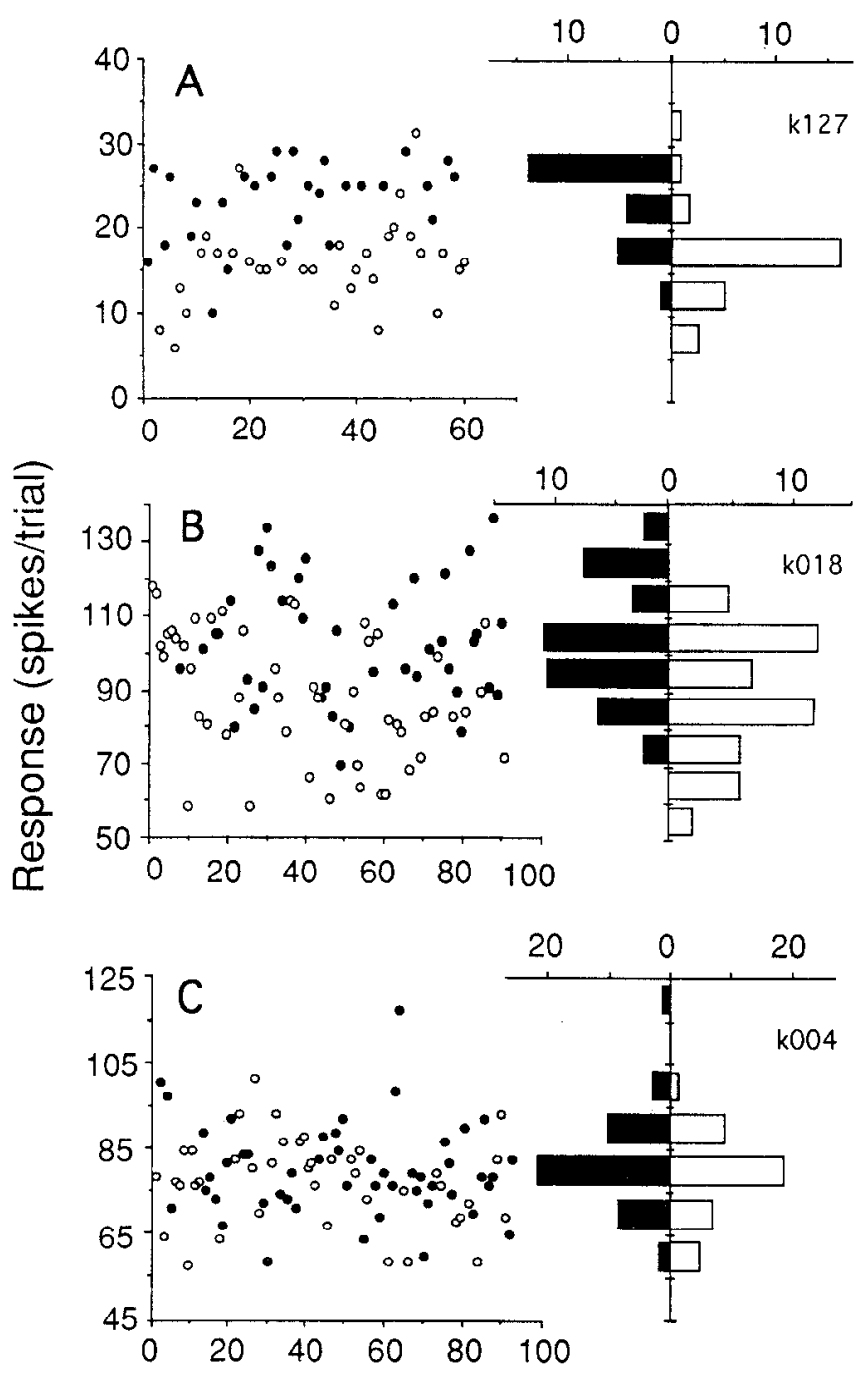

Trial number

Figure 9. Covariation of neuronal response and psychophysical decision (SOC effect). A, The scatterplot illustrates the responses of a single MST neuron to 60 presentations of a $0 \%$ correlation stimulus (pure motion noise). The solid circles indicate trials on which the monkey decided in favor of the neuron's preferred direction of motion (preferred decisions); the open circles indicate trials on which the monkey decided in favor of the null direction (null decisions). The data in the scatterplot are presented in the form of frequency histograms in the right panel. The ordinate indicates the response level (same scale as for the left panel) while the abscissa shows the frequency (number of trials) with which each response occurred. The histogram formed by the open bars was created by counting the open circles in the scatterplot within horizontally oriented bins. Similarly, the histogram formed by the solid bars was created by counting the solid circles within horizontally oriented bins. Both the scatterplot and the frequency histograms show that the monkey tended to choose the neuron's preferred direction when the neuron yielded a stronger than average response. Conversely, the monkey tended to choose the null direction when the neuron yielded a weaker response. The SOC value for this neuron was $0.85 . B$, The responses of a second MST neuron to 90 presentations of a $0 \%$ correlation stimulus. All conventions are the same as in $A$. The SOC value for this neuron was $0.70 . C$, The responses of a third neuron to 90 presentations of a $0 \%$ correlation stimulus. All conventions are the same as in $A$. The SOC value for this neuron was 0.54 . speeds substantially above or below this rangc (c.g., Newsome and Paré, 1988). Since the preferred speeds of many of our MST cells were well above this optimal range, we anticipated that psychophysical thresholds would be higher in these experiments, leading to the positive relationship between speed and threshold noted in the preceding paragraph. The new and interesting observation was that the neurons recorded in these experiments also yielded higher thresholds than neurons whose preferred speeds were within the optimal range for psychophysical performance. This dependence of threshold on speed is yet another interesting convergence between neuronal physiology and psychophysical performance on our task. We note in passing that scatterplots of neuronal versus psychophysical threshold for MT experiments (e.g., Britten et al., 1992, Fig. 10) do not show a strong relationship like that in Figure 8 , presumably because fewer MT neurons have preferred speeds outside the range of optimal psychophysical performance.

\section{Trial-by-trial correlation of neuronal response and psychophysical decision}

Multiple presentations of a particular, near-threshold stimulus will result in correct judgements of motion direction on some trials and incorrect judgements on others. Thus, performance on a discrimination task near psychophysical threshold reveals variability in the outcome of the psychophysical decision process. Similarly, the responses of individual neurons to a particular stimulus are not constant, but vary substantially from trial to trial about a mean response level appropriate for that stimulus condition. If MST contributes signals to the psychophysical decision process, it seemed possible that the substantial trial-totrial variability of a neuron's responses might have a measurable impact on the monkey's performance near threshold where decisions are likely to be most sensitive to fluctuations in the underlying neural signals. Specifically, we hypothesized that a larger than average response from a directionally selective neuron might increase the probability of a decision favoring that neuron's preferred direction. Our data permit us to address this question since we recorded both the response of an individual neuron and the monkey's psychophysical decision on a large number of trials involving stimuli near or below threshold. Remarkably, we observed such a covariation of neuronal response and psychophysical decision in MST and in a prior study of MT as well (Newsome et al., 1989; Britten et al., unpublished observations).

Figure 9 shows data from three different experiments to illustrate the range of effects found in MST. The three scatterplots depict the responses of three different MST neurons (ordinate) to repeated presentations of a $0 \%$ correlated stimulus (abscissa) As usual, the response was considered to be the total number of spikes occurring during the $2 \mathrm{sec}$ viewing interval. The data point for each trial is coded by the monkey's decision on that trial. Solid circles signify decisions in favor of the neuron's preferred direction, while open circles indicate decisions in favor of the null direction. In the experiment of Figure $9 A$, the mean response for the 60 illustrated trials was 19.3 spikes/trial, but the response varied considerably about the mean, as expected. The variation in the neuron's response from trial to trial was strikingly correlated with the monkey's decision. The monkey decided in favor of the preferred direction on $75 \%$ of the trials for which the neuron's response was greater than the mean; conversely, the monkey decided in favor of the null direction 
on $88 \%$ of the trials for which the response was less than the mean.

This effect is more conspicuous in the two frequency histograms in the right panel of Figure $9 A$. These histograms illustrate the distribution of neuronal responses obtained when the monkey decided in favor of the preferred direction (solid bars) and in favor of the null direction (open bars). The two histograms were formed simply by counting the appropriate data points in the scatterplot within horizontally oriented bins. Recall that all responses illustrated in Figure 9 were elicited by the same visual stimulus; the only difference between the two histograms is the monkey's decision concerning the direction of motion. The histograms confirm our impression from inspection of the scatterplot: the monkey decided in favor of the preferred direction far more frequently when the MST neuron responded strongly.

In Figure 9, $B$ and $C$ illustrate data from another two experiments in MST. In the experiment of Figure $9 B$, the monkey's decisions were correlated with the fluctuating responses of the MST neuron, though less conspicuously than in the experiment of Figure $9 A$. The response distribution for preferred decisions (solid bars) was clearly offset toward higher responses relative to the distribution for null decisions (open bars). In the experiment of Figure $9 C$, however, the monkey's decisions were only marginally related to the responses of the MST ncuron; the two response distributions were quite similar.

We quantified the covariation between neuronal response and psychophysical decision employing a method based on signal detection theory similar to that used to compute neurometric functions (see Methods and Materials). A receiver operating characteristic was computed for each pair of response distributions, and the normalized area under the ROC curve provided a nonparametric index of the effect (Newsome et al., 1989; Britten et al., unpublished observations). We refer to this index as a sender operating characteristic, or SOC value, because the difference in the two response distributions is not based on the stimulus received by the visual system, but on the decision made by the monkey that appears to result from signals sent by the cell.

The SOC value is bounded between zero and unity, but the valucs for most of our cclls fall betwecn 0.5 and unity. A valuc of unity would reflect a perfect correlation between the neuron's response and the monkey's decision; the monkey would always choose the preferred direction when the neuron's response was above some criterion level and would always choose the null direction when the response was below the criterion level. A value of 0.5 would indicate that the neuron's response and the monkey's decision are completely uncorrelated. Values significantly less than 0.5 would occur only in the counterintuitive case of anticorrelation between the neuron's response and the monkey's decision (i.e., weak neuronal responses lead to preferred decisions). In the experiment of Figure $9 A$, for example, the SOC value was 0.85 . The intermediate covariation evident in the experiment of Figure $9 B$ is reflected in the smaller SOC value of 0.70 , while much smaller covariation in Figure $9 C$ resulted in an SOC value of 0.54 .

The data in Figure 9 and the SOC values given in the preceding paragraph were based on trials with $0 \%$ correlated motion stimuli only. To obtain more trials and thus more statistical reliability in the SOC measurement, we computed an SOC value for each neuron based upon combined data from all stimulus conditions in which the monkey made at least three mistaken decisions (obviously, an SOC value cannot be computed if the

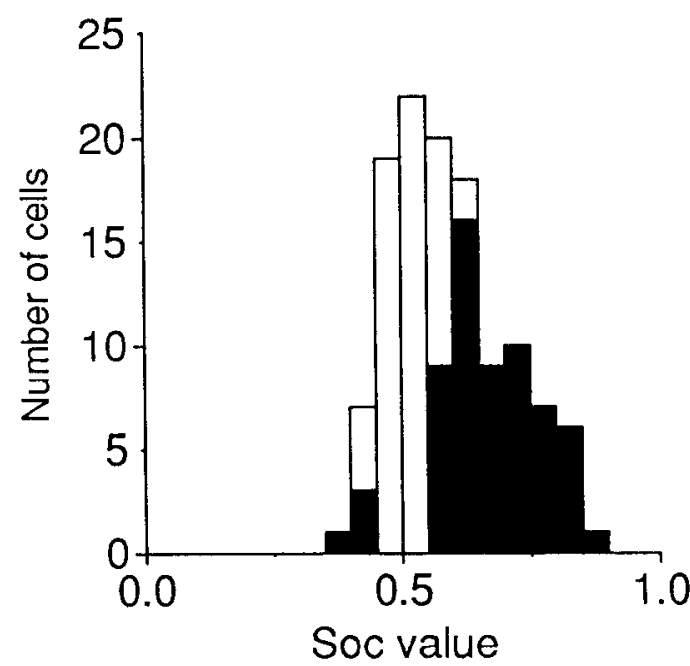

Figure 10. A frequency histogram of SOC values measured for the 120 MST neurons in our sample. Solld bars, representing $52 \%$ of the neurons in our sample, indicate SOC values that differed significantly from 0.5 .

monkey makes the same decision on every trial of a given motion condition). In order to eliminate the influence of the visual stimulus itself from the spike counts contributing to the SOC value, all spike counts were expressed in units of standard deviates by transforming the spike counts to $Z$ scores before data from the different stimulus conditions were combined. Combining the data in this manner is justified since prior analyses have shown that the SOC value does not vary systematically across stimulus conditions (Britten et al., unpublished observations). For each neuron, the $\mathrm{SOC}$ value thus obtained was evaluated for statistical significance by a comparison to its permutation distribution, generated under the null hypothesis that the neuronal response and psychophysical decision were randomly related. If the null hypothesis could be rejected, we concluded that the monkey's decisions were significantly correlated with the trial-to-trial fluctuations of the neuron's responses. (All of these analysis procedures will be described in detail by Britten et al. in a forthcoming publication.)

Figure 10 shows the distribution of SOC values computed for our entire sample of MST neurons. Values that differed significantly from 0.5 (the null hypothesis) are indicated by the solid bars while insignificant values are illustrated by the open bars. Fifty-two percent of the neurons in the sample yielded SOC values different from 0.5 , and the great majority of these were greater than 0.5 , indicating a positive correlation between neuronal response and psychophysical decision. Across the population as a whole, the mean SOC value was 0.594 , which was significantly larger than 0.5 ( $t$ test, $p<0.0001$ ). Thus, a trialto-trial covariation of neuronal response and psychophysical decision is not a rare observation, but appears to be characteristic of the population of MST neurons as a whole.

We next sought to establish the time course of the SOC effect in order to gain insight into possible underlying mechanisms (see Discussion). Specifically, we wondered whether the difference in cumulative spike counts (between preferred and null decision trials) resulted from a constant difference in firing rates throughout the $2 \mathrm{sec}$ visual stimulation interval or whether the tiring rates differed only during a restricted portion of the stimulation interval. In general, the firing rates of individual MST 


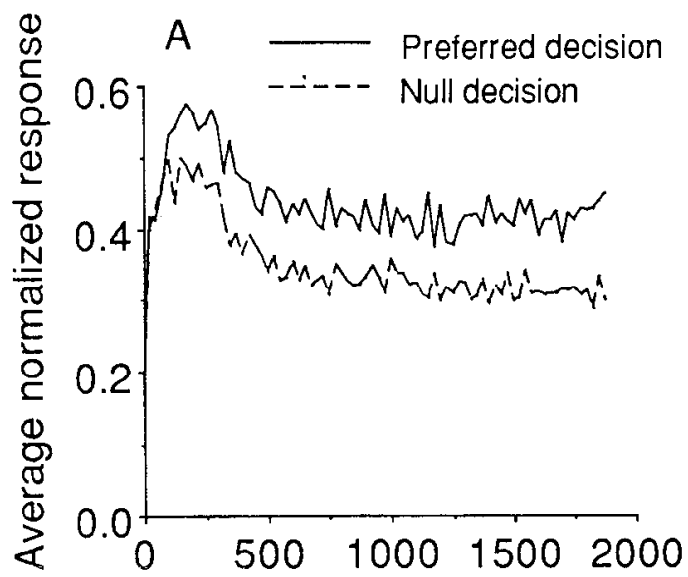

$B$

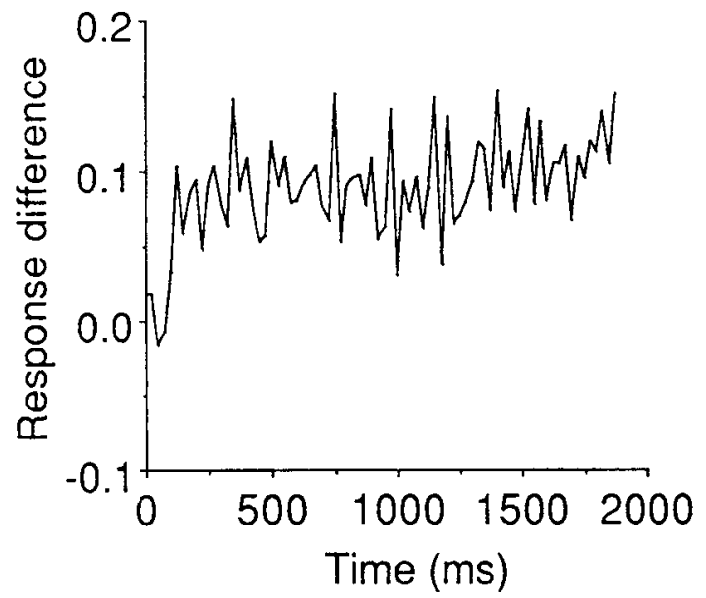

Figure 11. Time course of the SOC effect. A, Average responses are shown as a function of time within the 2 sec interval of visual stimulation. The solid line represents data for trials on which the monkey decided in favor of the preferred direction of motion; the dashed line depicts data for trials on which the monkey chose the null direction. Data from 57 MST neurons showing a significant SOC effect were normalized and averaged ( $0 \%$ correlation trials only). The bin size is 25 msec. $B$, Response difference as a function of time. The null decision data in $A$ are subtracted from the preferred decision data, and the difference is plotted bin by bin.

neurons are too low to permit a high resolution temporal analysis of the SOC effect. We therefore employed a method devised by Britten and colleagues (unpublished observations) to analyze poolcd data from all MST cclls that yielded an SOC effect significantly greater than 0.5 (57 cells; see Fig. 10). We analyzed data only from $0 \%$ correlation trials because this is the motion condition for which the monkey's decisions were most evenly divided between the preferred and null directions. Since absolute firing rates to the $0 \%$ stimulus varied enormously between cells, we normalized all responses before pooling the data. Normalization was accomplished by dividing the visual stimulation interval on each trial into consecutive $25 \mathrm{msec}$ bins and counting the number of spikes within each bin. The spike count in each bin was then normalized to the highest spike count encountered in any preferred decision trial for that particular neuron. Following normalization, the data from all 57 neurons were averaged on a bin-by-bin basis; averaging was performed separately for trials on which the monkey decided in favor of the preferred and null directions.

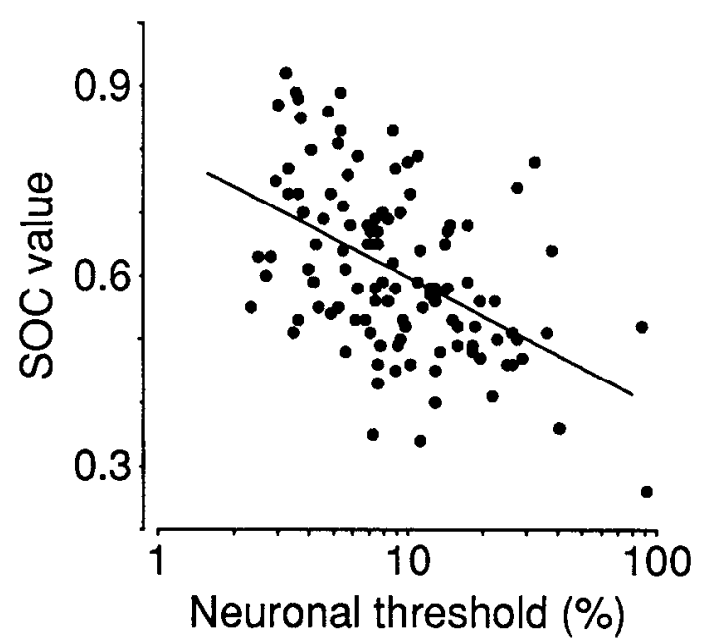

Figure 12. The relationship between the neuronal threshold $(\alpha)$ and SOC value for 120 MST neurons. The two measures are inversely related $(r=-0.47, p \leq 0.0001$, slope $=-0.17$, intercept $=0.75)$, indicating that the responses of sensitive neurons were better correlated with the monkey's decisions.

Figure $11 A$ shows the results of this analysis. Average normalized spike counts are displayed in $25 \mathrm{msec}$ bins covering the period of visual stimulation. The solid line indicates data for trials on which the monkey decided in favor of the cells' preferred direction, and the dashed line represents data for trials on which the monkey chose the null direction. Responses were essentially identical on the two sets of trials for the first $75 \mathrm{msec}$ of the response. At $100 \mathrm{msec}$, however, the responses diverged and remained well separated for the duration of the trial. Note that the normalized responses were calculated independently for each time bin, and the difference in firing rates was therefore present constantly after the first $100 \mathrm{msec}$ (i.e., the plotted responses are not cumulative). We infer that the difference in firing rates that gives rise to the $\mathrm{SOC}$ effect is present within the population of MST neurons for all but the first few bins of the response.

Figure $11 B$ shows the response difference between the two sets of trials plotted on a bin-by-bin basis. The difference appears to be roughly constant from $125 \mathrm{msec}$ through the first half to two-thirds of the response. The response difference may increase slightly, however, during the final $500 \mathrm{msec}$ of the trial. We tested this impression in two ways. First, a regression analysis of the data points from $200 \mathrm{msec}$ onward indicated a very weak, but significant, trend toward larger differences later in the trial ( $r=0.28$, slope $=0.017$ normalized response units per sec, $p$ $<0.02$ ). Second, a $t$ test showed that the spike counts in the last $825 \mathrm{msec}$ of the trial were significantly larger than the spike counts in the previous $825 \mathrm{msec}(p<0.05)$. Thus, the difference in firing rates appears to become slightly more pronounced as the trial proceeds.

\section{An inverse relationship between neuronal threshold and SOC value}

As documented in previous sections, both neuronal thresholds and SOC values vary broadly among the MST neurons. Interestingly, these two physiological characteristics appear to be related. Figure 12 shows neuronal threshold plotted against the $S O C$ value for each of the 120 neurons in our data set. The two measures are inversely correlated $(r=-0.47, p \leq 0.0001)$, 
indicating that the responses of an MST neuron are more likely to covary with the monkey's psychophysical decisions (high SOC values) if that neuron is very sensitive to the motion signals in the display (i.e., lower thresholds). This relationship withstood an analysis of covariance that controlled for possible confounding effects of independent variables such as monkey identity, aperture size, aperture eccentricity, and motion speed ( $p$ $<0.0001)$. Again, we will consider further the significance of this relationship in the Discussion.

\section{Comparison of MST and MT}

The data presented thus far demonstrate two basic relationships between neuronal and psychophysical performance: (1) an approximate identity between average neuronal threshold in MST and the average psychophysical threshold of our monkeys, and (2) a trial-to-trial covariation between neuronal response and perceptual decision for near-threshold stimuli. In both of these respects, our MST data are generally similar to MT data obtained from a group of three monkeys in a previous study (Newsome et al., 1989; Britten et al., 1992). To compare MST and MT precisely, however, it is desirable to obtain data from both areas in the same monkeys. Such data were readily available for one monkey that participated in both the prior study of MT and the present study of MST (monkey E, 52 MT neurons), and we have recently obtained MT data from the second monkey used in the present study (monkey K, 33 MT neurons). Since all of these experiments employed identical behavioral and visual stimulation techniques, we are able to compare MST and MT without the confounding influences of interanimal variability or disparate procedures.

The results of the MST and MT experiments were quite similar. The distribution of threshold ratios in Figure 6 did not differ significantly from the corresponding distribution for the 85 MT experiments ( $t$ test, $p=0.11$ ). In addition, the distribution of slope ratios was centered near unity for both areas (geometric mean $=1.13$ for MST, 0.93 for MT), although the small difference between the two areas approached statistical significance ( $t$ test, $p=0.06$ ). Using the statistical test for homogeneity outlined in Materials and Methods, we found that the neurometric function was indistinguishable from the psychometric function in roughly equal proportions of experiments in the two areas $(58.3 \%$ in MST; $62.4 \%$ in MT).

Since the ratios employed in Figures 6 and 7 only measure neuronal sensitivity relative to psychophysical sensitivity, we analyzed the neurometric data independently to determine whether MST neurons differed from MT neurons in their $a b$ solute sensitivity to motion signals in our displays. Across the entire data set, neuronal thresholds in MST were somewhat lower than those in MT ( $t$ test, $p<0.01$ ), but this improvement could be explained by other independent variables that differed between the two sets of experiments. An analysis of covariance that controlled for the influences of these independent variables (aperture size, eccentricity, speed of the motion signal, and monkey identity) showed no effect of area identity on neurometric thresholds ( $p=0.23)$. Unfortunately, the issue of absolute neuronal sensitivity remains somewhat ambiguous bccause the sampled range of eccentricities and aperture sizes differed substantially between MST and MT (recall that we tailored the visual stimulus as nearly as possible to the preference of each neuron studied). Thus, our analysis of covariance involved an extrapolation between data sets with relatively little overlap of key independent variables, and it remains possible that neuronal

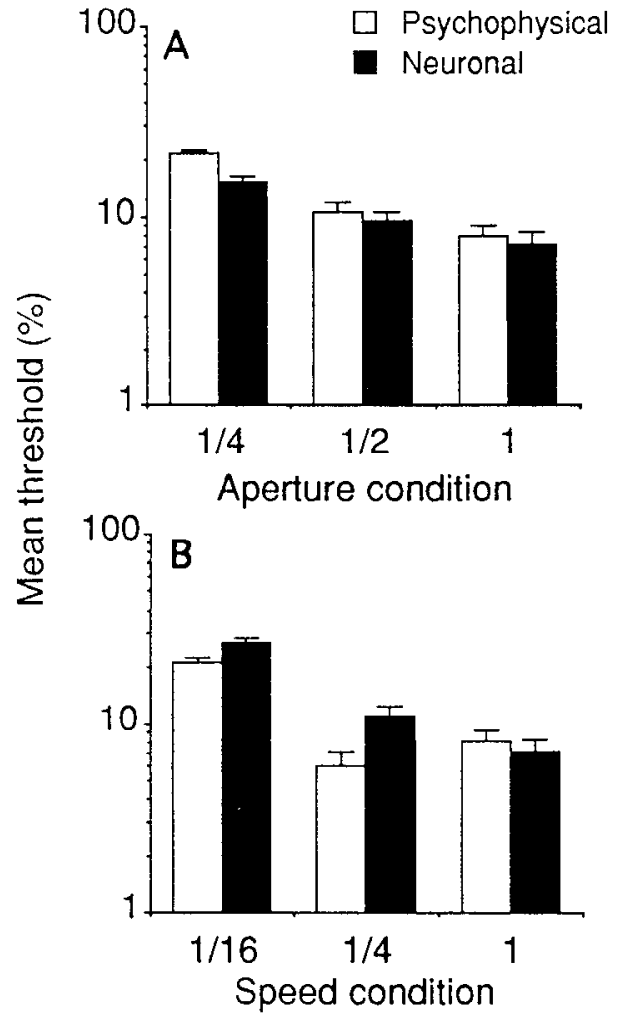

Figure 13. Influence of nonoptimal stimulus conditions on psychophysical and neuronal thresholds (open bars and solid bars, respectively). Thresholds are given as geometric means with associated error bars. $A$, Influence of aperture size. Three conditions were tested: an aperture diameter as close as possible to optimal $(I), 50 \%$ of optimal $(I / 2)$, or $25 \%$ of optimal $(1 / 4)$. All other stimulus parameters were held constant. $B$, Influence of stimulus speed. Three conditions were tested: $(l)$ the optimal speed for the neuron, $25 \%$ of optimal $(1 / 4)$, and $6.25 \%$ of optimal (1/16). All other parameters were held constant.

thresholds in MST and MT might differ somewhat if tested with precisely identical aperture sizes, eccentricities, and motion speeds. Nevertheless, our data suggest that any difference in absolute sensitivity between the two areas is likely to be small.

MST neurons also conformed closely to MT neurons in exhibiting a trial-to-trial covariation of response magnitude and psychophysical decision. The mean SOC value in our sample of MT neurons was 0.58 as compared to a mean of 0.59 in MST, and the distributions of SOC values obtained in the two areas were indistinguishable ( $t$ test, $p=0.28$ ). In addition, our sample of MT neurons yielded the same inverse relationship between SOC value and neuronal threshold illustrated for MST in Figure 12 (correlation coefficient $=-0.52, n=85, p<0.0001$ ).

\section{Influence of nonoptimal stimulus conditions}

In all experiments presented thus far, we created optimal conditions for observing congruence between neuronal and psychophysical performance by matching the visual stimulus conditions as closely as possible to the physiological propertics of the neuron under study. It seemed possible, however, that these relationships might be disrupted if the visual stimulus conditions differed substantially from the optimal configuration for individual MST neurons. In the following experiments, we addressed this question by measuring neuronal and psychophysical performance first under optimized stimulus conditions and then 


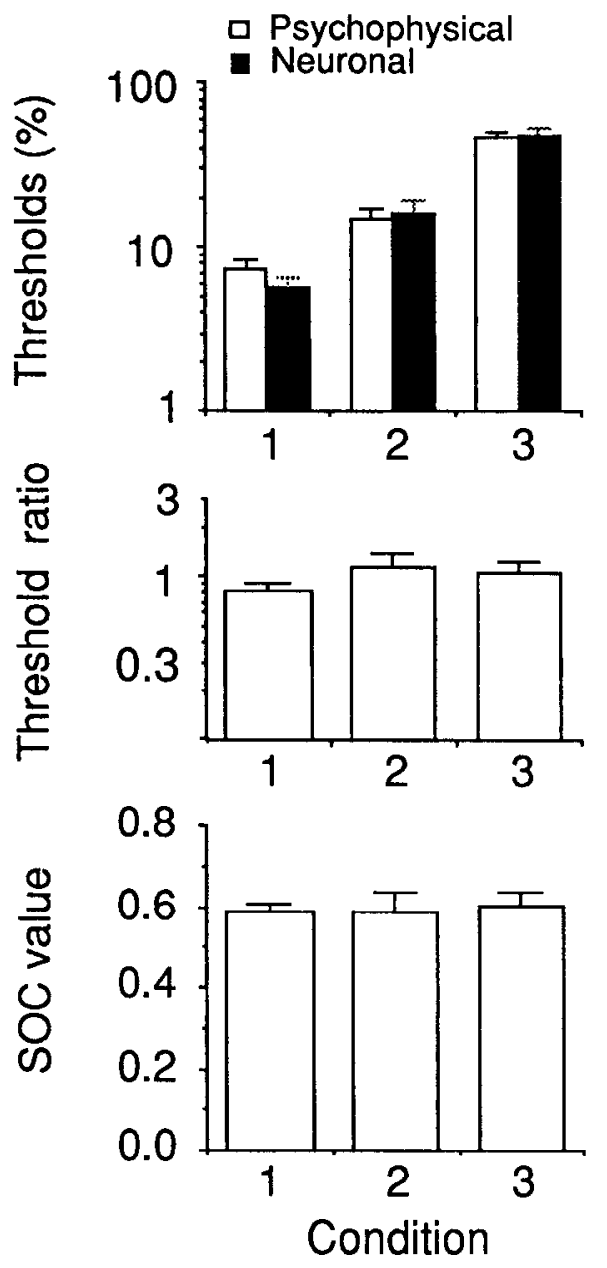

Figure 14. Psychophysical and neuronal performance when aperture size and speed are changed simultaneously. Top. Psychophysical and neuronal thresholds for three stimulus conditions: optimal speed and aperture size (1), $25 \%$ of optimal speed and $25 \%$ of optimal aperture diameter (2), $6.25 \%$ of optimal speed and $12.5 \%$ of optimal aperture diameter (3). Thresholds are given as geometric means with associated error bars. Middle, Ratios of neuronal threshold to psychophysical threshold for the three test conditions described in the top panel. Threshold ratios are given as geometric means with associated error bars. Bottom, Mean SOC values for the three test conditions described in the top panel.

under test conditions in which mismatches were introduced along two stimulus dimensions-aperture size and motion speed.

In the first set of experiments we reduced the stimulus aperture size while holding the speed and the axis of the motion signal constant at the optimal values for the neuron under study. For each neuron, we attempted to test the "best" aperture size (the control condition) as well as two reduced apertures whose diameters were one-half and one-fourth of the "best" diameter. The control condition was tested on 36 neurons, but isolation was lost on some neurons before both test conditions could be completed. Thus, 25 neurons were tested with the one-half diameter aperture and 27 neurons with the one-fourth diameter aperture. Because MST receptive fields are typically very large, the "best" aperture was almost always $30^{\circ}$ in diameter, the maximum achievable with our hardware configuration (see Materials and Methods). The eccentricity of the center of the aperture was kept constant as the size was varied.

Figure $13 A$ shows the results of these experiments. Neuronal

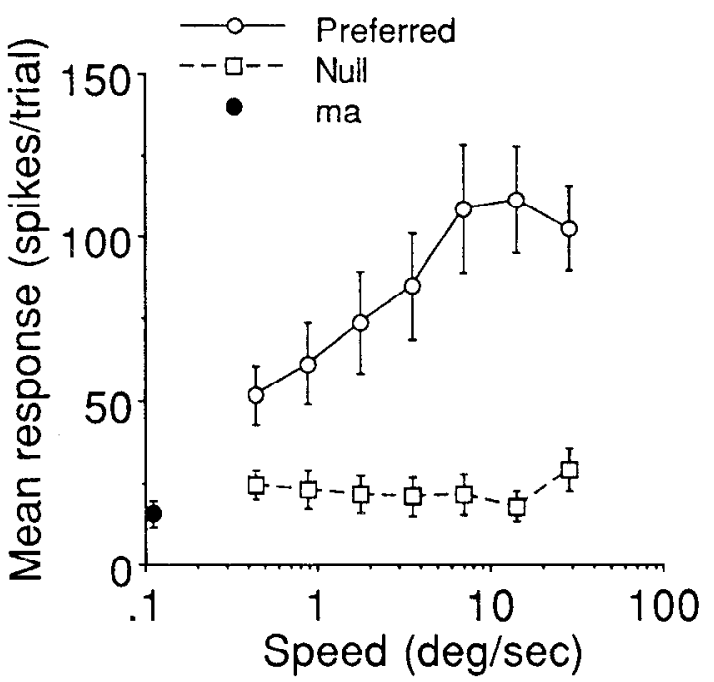

Figure 15. Mean response as a function of motion speed for 14 MST neurons. The open circles and the solid line represent responses to preferred direction motion; the open squares and the dashed line illustrate responses to null direction motion. The solid circle shows mean maintained activity for the 14 neurons. On average, the MST neurons responded well and were directionally selective across the entire range of speeds tested.

thresholds increased as the stimulus aperture was reduced in size, but psychophysical thresholds were affected in a similar fashion so that the approximate identity of the two measures was unchanged by the manipulation. This impression was confirmed by a one-way ANOVA performed on the log transformed ratios of neuronal to psychophysical threshold. Threshold ratios were unaffected by aperture size $(p=0.10)$.

We next examined the effect of varying motion speed while holding other stimulus variables constant. For 12 MST neurons we attempted to test the optimal speed (the control condition) as well as two reduced speeds: one-fourth of the optimal and $1 / 16$ of the optimal. We obtained satisfactory data at one-fourth of the optimal speed for 10 cells, and at 1/16 of the optimal speed for nine cells. Across the sample of cells, the speeds tested ranged from $0.4 \mathrm{degree} / \mathrm{sec}$ to 28.4 degrees/sec. Again, Figure $13 B$ shows that neuronal and psychophysical thresholds were affected similarly by this manipulation, the general trend being an increase in threshold as stimulus speed decreased from the optimal. A one-way ANOVA demonstrated a weakly significant effect of speed on threshold ratios (logarithmically transformed, $p=0.046$ ), and a test of contrasts showed that this difference resulted from a discrepancy between neuronal and psychophysical thresholds when motion speed was one-quarter of the optimal (Fig. 13B). This result is probably misleading, however, because no discrepancy was evident for the more stringent speed reduction (1/16 of optimal; Fig. 13B) or for the combined aperture and speed reductions in the final set of experiments described below.

Since the equality of neuronal and psychophysical thresholds was little affected by the manipulations described thus far, we conducted a final set of experiments in which both aperture size and motion speed were changed simultaneously. Aperture size and speed were changed in a yoked fashion (slower speeds for smaller apertures) to mimic stimulus conditions that are likely to be more appropriate for neurons at earlier stages of the motion pathway. In Figure 14, condition 1 refers to the control condition 
in which the stimulus aperture was large $\left(30^{\circ}\right.$ diameter) and the motion speed was optimal for the cell under study ( 16 cells tested). In condition 2 , the speed was reduced to a quarter of the optimal while the diameter of the aperture was reduced to $7.5^{\circ}$ ( 13 cells tested). In condition 3 , the speed was further reduced to $1 / 16$ of optimal while the diameter of the aperture was reduced to $3.75^{\circ}$ (nine cells tested).

The top panel of Figure 14 shows that neuronal and psychophysical thresholds remained approximately equal, on average, even for the most drastic stimulus manipulation. Thresholds increased by more than a factor of five between conditions 1 and 3 , but the increases were proportionally similar for the neurons and the psychophysical observers. The middle panel of Figure 14 illustrates mean threshold ratios (log transformed) for each of the three test conditions, and a one-way ANOVA confirmed that these threshold ratios were not significantly affected by stimulus condition $(p-0.41)$.

The bottom panel of Figure 14 shows that SOC values were unaffected by manipulations of aperture size and motion speed. The average SOC value appeared remarkably constant across these experiments, and a one-way ANOVA substantiated this impression $(p=0.92)$. Thus, the trial-to-trial covariation of neuronal response and psychophysical decision is also robust in the face of marked stimulus manipulations.

Finally, we note that thresholds for MST neurons could have been elevated trivially at very low speeds if the neurons were simply unresponsive in this speed range. Our qualitative impression was that MST neurons were surprisingly responsive to very low speeds, but we measured speed tuning curves quantitatively for 14 neurons in order to test our impression. Figure 15 shows the averaged responses of these 14 neurons for preferred (circles) and null (squares) directions of motion over speeds ranging from 0.4 to 28.4 degrees $/ \mathrm{sec}$. While optimal speeds were in the range of 10-30 degrees/sec, MST neurons were, on average, both responsive and directionally selective at the lowest speeds employed in this study. Thus, the elevated neuronal thresholds in Figure $13 B$ do not simply reflect a lack of responsiveness.

\section{Discussion}

In this study we compared the ability of rhesus monkeys and single MST neurons to discriminate the direction of motion in a stochastic random dot display. Average psychophysical and neuronal thresholds, measured on the same trials under precisely identical conditions, proved to be remarkably similar. In addition, the slopes of the psychometric and neurometric functions relating discriminative ability to the strength of the motion signal were nearly the same on average. Thus, single MST neurons carry motion signals that are well matched to the psychophysical performance of our monkeys.

We are confident for several reasons that this convergence of neuronal and psychophysical data is not artifactual. First, the behavior of our monkeys was under good stimulus control throughout these experiments as indicated by excellent asymptotic performance levels (reliably at or near $100 \%$ correct for strong motion signals) and by the consistent slopes of the psychometric functions. Poor performance due to inattention or satiation would result in deterioration of both of these features of the psychometric data. Thus, psychophysical thresholds were not elevated artificially because of poor behavioral control. Second, our comparison of neuronal and psychophysical performance is not corrupted by a mismatch between neuronal and psychophysical integration times. Our computation of neuronal thresholds is based on the assumption of a $2 \mathrm{sec}$ integration time, since the neuronal response was considered to be the integrated spike count over the full $2 \mathrm{sec}$ stimulus presentation interval. This assumption is justified by psychophysical evidence that our monkeys accumulate information throughout the $2 \mathrm{sec}$ viewing interval in reaching their decisions (Britten et al., 1992). Thus, spike counts integrated over the full $2 \mathrm{sec}$ viewing interval are appropriate for comparing neuronal to psychophysical performance on our task.

In addition to the general similarity of neurometric and psychometric functions, we found that psychophysical decisions concerning the direction of motion were significantly correlated with the variable trial-to-trial responses of many MST neurons. For repeated presentations of a near-threshold stimulus, the monkey was more likely to choose the neuron's preferred direction of motion on trials in which that neuron yielded a larger than average response. In other words, a measurable portion of the variance in the psychophysical decision process could be attributed to the response variance of single neurons in MST.

In a prior study of MT, several control experiments were conducted to rule out various trivial explanations for this effect (Britten et al., unpublished observations). For example, variance can be eliminated from the stochastic stimulus itsclf by presenting precisely identical sequences of dots for each presentation of a particular motion condition. This manipulation had no measurable effect on the covariation between neuronal response and psychophysical decision, thus suggesting a neural origin for the effect. Assuming a neural origin, at least two explanations for the effect come immediately to mind. Most straightforward, trial-to-trial variability in neuronal response could influence the outcome of threshold decisions if the MST neuron under study contributes information to the monkey's decision process. Alternatively, the activity of MST neurons could be modulated by feedback from higher centers after the decision is made. Either scenario would lead to a covariation of neuronal response and the monkey's decision.

To test the latter possibility, we examined the time course of the SOC effect during the $2 \mathrm{sec}$ stimulus presentation interval. A response difference between preferred and null decision trials arising late in the $2 \mathrm{sec}$ stimulation interval would be consistent with the notion that responses in MST are enhanced by feedback after the decision is made. On the other hand, a response difference arising early in the stimulation interval is less likely to result from a feedback mechanism since previous psychophysical data show that our monkeys integrate information over a large portion of the $2 \mathrm{sec}$ stimulus presentation interval in order to reach their decisions (Britten et al., 1992). The data in Figure 11 show clearly that the SOC effect arises at nearly its full amplitude $100 \mathrm{msec}$ into the stimulation period. We therefore suggest that the principal cause of covariation between neuronal response and psychophysical decision is that variability in the responses of sensory neurons influences the outcome of the monkey's decisions. As indicated in Results, the SOC effect in MST neurons appears to be slightly larger in the second half of the visual stimulation interval, and it therefore remains possible that some small portion of the effect may result from feedback after the decision is made. Alternatively, the monkey's decision process may place more weight on recently accrued information, leading to a somewhat larger SOC effect late in the trial.

Given the large numbers of cortical neurons that respond to any given visual stimulus, it is surprising that the responses of 
single cortical neurons appear to have a measurable influence on psychophysical decisions. Recent experimental and modeling efforts in our laboratory have produced a plausible solution to this conundrum. Briefly, the effect can be explained by the presence of correlated responses within the pool of sensory neurons contributing to the psychophysical decision process. In an cxtensive set of Monte Carlo simulations, weakly correlated responses like those observed in multiunit recordings in MT (Zohary et al., 1992) gave rise to a trial-to-trial covariation of neuronal response and psychophysical decision of similar magnitude to that observed in the present study and in a prior study of MT (Shadlen et al., 1992). At an intuitive level, the effect arises in our simulations because a fraction of the trial-to-trial response variance is correlated among all neurons in the pool that contribute to the decision. Although this fraction is small, it has a measurable impact on performance near or below psychophysical threshold where the outcome of a decision hinges upon small fluctuations in the underlying signals. Thus, the covariation of neuronal response and psychophysical decision can be regarded as a signature of the monkey's use of neuronal signals in reaching his decision. A similar trial-to-trial covariation betwecn neuronal response and psychophysical decision has been reported in nociceptive pathways of the somatosensory system by Dubner and colleagues (Kenshalo et al., 1988; Dubner et al., 1989), raising the intriguing possibility that this effect will prove to be a generally useful method for identifying populations of neurons that contribute information to specific psychophysical discriminations.

Interestingly, the two physiological properties emphasized in our analysis - threshold and the covariation with psychophysical decision - are themselves related. The responses of sensitive neurons (lower thresholds) are better correlated with the monkeys' psychophysical decisions, as indicated by their higher SOC values (Fig. 12). If the covariation with psychophysical decision (denoted by the SOC value) arises, as we suggest, from correlated response fluctuations within the pools of sensory neurons, then the data in Figure 12 imply that sensitive neurons are likely to be more strongly intercorrelated than insensitive neurons. In fact, a preliminary analysis of multiunit data from MT suggests that this implication is true (Zohary et al., unpublished observations). The relationship in Figure 12 can be reproduced in Monte Carlo simulations by incorporating stronger intercorrelation between more sensitive input neurons (Shadlen et al., unpublished observations).

\section{Nonoptimal stimulus conditions}

In our prior comparisons of neuronal and psychophysical performance, we have tailored our visual stimuli to match the optimal physiological propertics of the neuron under study. The reasons for this approach were twofold. First, different classes of neurons are likely to play more or less important roles in mediating psychophysical performance, depending upon the nature of the task. Thus, it seemed sensible to analyze neuronal activity under conditions in which that activity is particularly relevant to the psychophysical problem at hand. Second, we felt that relating neuronal sensitivity to psychophysical performance would be more straightforward if the neuron and the observer had equal access to the visual stimulus.

From this perspective, our examination of nonoptimal stimulus conditions in the present study was revealing and somewhat surprising. Although absolute thresholds increased under nonoptimal conditions, we found that the ratio of neuronal to psy- chophysical threshold remained near unity despite reduction of the stimulus aperture diameter to $12.5 \%$ of optimal and the stimulus speed to $6.25 \%$ of optimal. Thus, MST neurons carry signals appropriate for mediating psychophysical performance across an impressively broad range of aperture sizes and speeds. Consistent with this observation, the trial-to-trial covariation of neuronal response and psychophysical decision (SOC value) also was unaffected by our stimulus manipulations, suggesting that MST neurons actually influence psychophysical performance under the altered conditions.

While our evidence implies that individual MST neurons contribute to performance over a very broad range of speeds and aperture sizes, we suspect that any cell's contribution is more sharply limited as the axis of motion is changed. We did not manipulate this parameter in the present study, but it is easy to intuit that neuronal thresholds should rise precipitously as the axis of motion becomes orthogonal to the preferred since most neurons will respond similarly to both directions of motion along the orthogonal axis. This intuition has been confirmed by recent experiments in MT showing that neuronal thresholds increase by an average of $70 \%$ for axes of motion that differ from the preferred by $30^{\circ}$ (Britten and Newsome, 1993). In addition, we have recently observed that the SOC values of MT neurons fall significantly when the monkey performs the direction discrimination along an axis of motion orthogonal to the preferred, suggesting that these neurons have little influence on such discriminations (Britten et al., unpublished observations). Thus, both major correspondences between neuronal and psychophysical performance break down at the orthogonal axis of motion, suggesting that the sensory signals supporting psychophysical performance along the orthogonal axis arise from a different population of cells. Together, these considerations imply that MST neurons contribute sensory signals to the decision process over a very broad range of aperture sizes and speeds as long as the motion occurs along or reasonably near their preferred-null axes. If the direction of motion departs substantially from the preferred-null axis, however, a population of neurons more closcly tuncd to the actual dircction of motion is likely to exert a stronger influence on the monkey's decision. This conclusion seems reasonable in light of the fact that our monkeys perform extended blocks of trials in which the axis of motion is kept constant during study of a particular neuron. Performance would presumably benefit if the decision process admitted information selectively from columns of neurons whose preferred-IIull axes are reasonably matched to the intended axis of discrimination.

It is interesting to note that the most extreme stimulus configurations employed in this study $\left(3.75^{\circ}\right.$ diameter apertures, $0.5-3.0$ degree/sec speeds) would likely correspond to the receptive field sizes and optimal speed ranges of many V1 neurons at comparable eccentricities $\left(15-30^{\circ}\right)$. If directionally selective neurons in $\mathrm{Vl}$, like those in $\mathrm{MSl}$ and $\mathrm{Ml}$, yielded neuronal thresholds equivalent to psychophysical threshold for these stimuli, we would be forced to conclude that information encoded by V1 neurons can be transmitted in a relatively unmodified manner through intervening levels of the motion pathway to MST. In other words, the responses of neurons at several levels of the motion pathway to roughly optimal stimulus configurations may be reflected in the responses of MST neurons to reduced stimulus configurations. Such an arrangement would be convenient in the context of our psychophysical paradigm because the decision process could operate efficiently by sam- 
pling sensory information only from later stages of the motion pathway. In future experiments we will examine the activity of VI and V3 neurons during performance on our task to gain insight into such issues.

In light of the present results, how critical is our procedural stipulation that stimulus properties be well matched to receptive field properties in experiments of this nature? The preceding discussion indicates that our basic conclusions would be substantially unaffected by mismatches in the domain of stimulus speed and aperture size, as long as the neuron and the psychophysical observer have access to the same information. The data in Figures 13 and 14, for example, show that our comparison of neuronal to psychophysical sensitivity would have been inaccurate had the neuron been analyzed with one aperture size or motion speed and psychophysical performance with another. As a practical matter, stimulus apertures should always be equal to or smaller in size than the receptive field to estimate accurately the relative sensitivities of single neurons and psychophysical observers. Larger apertures give the psychophysical observer an unfair advantage in sensitivity by permitting spatial pooling across different receptive field locations (Hawken and Parker, 1990). Presumably, a single neuron has no "access" to portions of the stimulus outside its receptive field boundaries, and may in fact be inhibited by suppressive surround mechanisms (Allman et al., 1985).

In the domain of motion direction, the relation of neuronal to psychophysical performance differs substantially with the axis of motion even if the neuron and the observer have equal access to the stimulus. In general, then, matching visual discriminanda to receptive field properties is important for estimating reliably the relative sensitivities of neurons and psychophysical observers. Departures from this approach can be very informative, however, for asking specific questions about the possible contribution of neurons to particular modifications of a psychophysical task.

\section{Transmission of signals within the motion pathway}

Several laboratories have shown that MST contains sophisticated circuitry that processes motion signals concerning optic flow (Tanaka et al., 1986; Andersen et al., 1990; Duffy and Wurtz, 1991). This circuitry appears to be invisible, however, from the point of view of the simple motion signals carried by MST neurons during performance on our psychophysical task. MST neurons possess nearly the same sensitivity to motion as do their MT afferents and thus appear to transmit these signals faithfully to higher level circuits which presumably form and implement the psychophysical decision. High-fidelity transmission of neural signals is not a trivial accomplishment since afferent signals can be degraded by noise sources at each level of the pathway. We suspect that pooling of afferents from MT and other areas generates an increase in sensitivity that offsets the losses resulting from intrinsic noise sources within MST. From this point of view, faithful transmission of motion signals within the visual cortex is not simply a passive process but requires active protection against noise degradation. Our laboratory is presently engaged in a modeling exercise to gain quantitative insight into the sources of neural noise and into the nature of the pooling mechanisms that may ameliorate the effects of noise.

\section{References}

Albright TD (1993) Cortical processing of visual motion. In: Visual motion and its role in the stabilization of gaze (Miles FA, Wallman J, eds), pp 177-201. New York: Elsevier.
Albright TD, Desimone R (1987) Local precision of visuotopic organization in the middle temporal area (MT) of the macaque. Exp Brain Res 65:582-592.

Allman JM, Meizin F, McGuinness E (1985) Direction and velocityspecific responses from beyond the classical receptive field in the middle temporal visual area (MT). Perception 14:105-126.

Andersen R, Snowden R, Treue S, Graziano M (1990) Hierarchical processing of motion in the visual cortex of monkey. In: Cold Spring Harbor symposia on quantitative biology, Vol LV, The brain, pp 741-748. Plain View, NY: Cold Spring Harbor Laboratory.

Barlow HB, Levick WR, Yoon M (1971) Responses to single quanta of light in retinal ganglion cells of the cat. Vision Res [Suppl] 3:87101.

Bradley A, Skottun BC, Ohzawa I, Sclar G, Freeman RD (1987) Visual orientation and spatial frequency discrimination: a comparison of single cells and behavior. J Neurophysiol 57:755-772.

Britten KH, Newsome WT (1993) The relationship between directional tuning and motion strength in area MT. Soc Neurosci Abstr 19:1282.

Britten KH, Shadlen MN, Newsome WT, Movshon JA (1992) The analysis of visual motion: a comparison of neuronal and psychophysical performance. J Neurosci 12:4745-4765.

Britten KH, Shadlen M, Movshon JA, Newsome WT (1993) The responses of MT neurons to variable strength stochastic motion signals. Vis Neurosci 10:1157-1169.

Celebrini S, Newsome WT (1992) Responses of neurons in area MST during direction discrimination performancc: a comparison of ncuronal and psychophysical sensitivity. Soc Neurosci Abstr 18:1101.

Cohn TE, Green DG, Tanner WP (1971) Receiver operating characteristic analysis. Application to the study of quantum fluctuation in optic nerve of Rana pipiens. J Gen Physiol 66:583-616.

Crist CF, Yamasaki DSG, Komatsu H, Wurtz RH (1988) A grid system and a microsyringe for single cell recording. J Neurosci Methods $26: 117-122$.

Dean AF (1981) The variability of discharge of simple cells in cat striate cortex. Exp Brain Res 44:437-440.

Desimone R, Ungerleider LG (1986) Multiple visual areas in the caudal superior temporal sulcus of the macaque. J Comp Neurol 248: 164-189.

Dubner R, Kenshalo DR, Maixner W, Bushnell MC, Oliveras JL (1989) The correlation of monkey medullary dorsal horn neuronal activity and the perceived intensity of noxious heat stimuli. J Neurophysiol 62:450-457.

Duffy CJ, Wurtz RH (1991) Sensitivity of MST neurons to optic flow stimuli. I. A continuum of response selectivity of large-field stimuli. J Neurophysiol 65:1329-1345.

Evarts EV (1968) Methods for recording activity of individual neurons in moving animals. Methods Med Res 11:241-250.

Felleman D, Van Essen D (1991) Distributed hierarchical processing in the primate cerebral cortex. Cereb Cortex 1:1-47.

Green DM, Swets JA (1966) Signal detection theory and psychophysics. New York: Wiley.

Hawken MJ, Parker AJ (1990) Detection and discrimination mechanisms in the striate cortex of the Old-World monkey. In: Vision: coding and efficiency (Blakemore $\mathrm{C}$, ed), pp 103-116. Cambridge: Cambridge UP.

Judge SJ, Richmond BJ, Chu FC (1980) Implantation of magnetic search coils for measurement of eye position: an improved method. Vision Res 20:535-538.

Kenshalo DR, Chudler EH, Anton F, Dubner R (1988) SI nociceptive neurons participate in the encoding process by which monkeys perceive the intensity of noxious thermal stimulation. Brain Res 454: 378-382.

Komatsu H, Wurtz R (1988) Relation of cortical areas MT and MST to pursuit eye movements. I. Localization and visual properties of neurons. J Neurophysiol 60:580-603.

Maunsell JHR, Newsome WT (1987) Visual processing in monkey extrastriate cortex. Annu Rev Neurosci 10:363-401.

Maunsell JHR, Van Essen DC (1983) The connections of the middle temporal visual area (MT) and their relationship to a cortical hierarchy in the macaque monkey. J Neurosci 3:2563-2586.

Maunsell JHR, Van Essen DC (1987) Topographic organization of the middle temporal visual area in the macaque monkey: representational biases and the relationship to callosal connections and myeloarchitectonic boundaries. J Comp Neurol 266:535-555.

Murasugi CM, Salzman CD, Newsome WT (1993) Microstimulation 
in visual area MT: effects of varying pulse amplitude and frequency. J Neurosci 13:1719-1729.

Newsome WT, Paré EB (1988) A selective impairment of motion perception following lesions of the middle temporal visual area (MT). J Neurosci 8:2201-2211.

Newsome WT, Wurtz RH, Komatsu H (1988) Relation of cortical areas MT and MST to pursuit eye movements. II. Differentiation of retinal from extraretinal inputs. J Neurophysiol 60:604-620.

Newsome WT, Britten KH, Movshon JA, Shadlen M (1989) Single neurons and the perception of visual motion. In: Neural mechanisms of visual perception. Proceedings of the retina research foundation (Lam DM-K, Gilbert CD, eds), pp 171-198. The Woodlands, TX: Portfolio.

Quick RF (1974) A vector magnitude model of contrast detection. Kybernetik 16:65-67.

Saito H, Yukie M, Tanaka K, Hikosaka K, Fukada Y, Iwai E (1986) Integration of direction signals of image motion in the superior temporal sulcus of the macaque monkey. I Neurosci 6:145-157.

Salzman CD, Britten KH, Newsome WT (1990) Cortical microstimulation influences perceptual judgements of motion direction. Nature 346:174-177.

Salzman CD, Murasugi CM, Britten KH, Newsome WT (1992) Microstimulation in visual area MT: effects on direction discrimination performance. J Neurosci 12:2331-2355.

Shadlen MN, Newsome WT, Britten KH, Zohary E, Movshon JA (1992) Predicting psychophysical performance from pooled neuronal responses. Soc Neurosci Abstr 18:1101.

Snowden RJ, Treue S, Andersen RA (1992) The response of neurons in areas V1 and MT of the alert rhesus monkey to moving random dot patterns. Exp Brain Res 88:389-400.
Tanaka K, Saito H (1989) Analysis of motion of the visual field by direction, expansion/contraction and rotation cells clustered in the dorsal part of the medial superior temporal area of the macaque monkey. J Neurophysiol 62:626-641.

Tanaka K, Hikosaka H, Saito H, Yukie Y, Fukada Y, Iwai E (1986) Analysis of local and wide-field movements in the superior temporal visual areas of the macaque monkey. J Neurosci 6:134-144.

Tolhurst DJ, Movshon JA, Dean AF (1983) The statistical reliability of signals in single neurons in cat and monkey visual cortex. Vision Res 23:775-785.

Ungerleider LG, Desimone R (1986) Cortical connections of visual area MT in the macaque. J Comp Neurol 248:190-222.

Van Essen DC, Maunsell JHR, Bixby JL (1981) The middle temporal visual area in the macaque: myeloarchitecture, connections, functional properties and topographic representation. J Comp Neurol 199: 293-326.

Vogels R, Orban GA (1990) How well do response changes of striate neurons signal differences in orientation: a study in the discriminating monkey. J Neurosci 10:3543-3558.

Vogels R, Spileers W, Orban GA (1989) The response variability of striate cortical neurons in the behaving monkey. Exp Brain Res 77: $432-436$.

Watson AB (1979) Probability summation over time. Vision Res 19: $515-522$.

Zeki S (1991) Cerebral akinetopsia (visual motion blindness). Brain 114:811-824.

Zohary E, Hillman P, Hochstein S (1990) Time course of perceptual discrimination and single neuron reliability. Biol Cybern 62:475-486.

Zohary E, Shadlen MN, Newsome WT (1992) Correlated activity of neurons in area MT. Soc Neurosci Abstr 18:1101. 